\title{
Ambulante Jugendhilfe: Ein systematisches Literatur-Review zur Arbeits- und Gesundheitssituation
}

\author{
Julia C. Lengen ${ }^{1} \cdot$ Maren Kersten $^{2} \cdot$ Sabine Gregersen $^{2}$
}

Angenommen: 9. Dezember 2020 / Online publiziert: 28. Dezember 2020

(c) Der/die Autor(en) 2020

\section{Zusammenfassung}

Das vielfältige Tätigkeitsfeld der Jugendhilfe im ambulanten Bereich ist von diversen - unter anderem psychischen Einflussfaktoren geprägt, die sich auf die Gesundheit der pädagogischen Fachkräfte auswirken können.

Die Zusammenhänge zwischen den gesundheitsförderlichen (Ressourcen) sowie gesundheitsgefährdenden (Stressoren) psychischen Belastungsfaktoren und der psychischen Gesundheit sollen mit Hilfe einer systematischen Literaturrecherche betrachtet werden. Nach dem PEO-Schema wird der Forschungsfrage im Zeitraum 2000-2018 in den Datenbanken Medline, PsyInfo und Psyndex nachgegangen, um einen Überblick über die Arbeits- und Gesundheitssituation der Zielgruppe zu erlangen.

Eine Vielzahl von Stressoren und Ressourcen, vor allem in den Bereichen Arbeitsinhalt, Arbeitsorganisation und soziale Beziehungen stehen in einem Zusammenhang mit der psychischen Gesundheit der Beschäftigten. Starke bis mittlere Zusammenhänge weisen die Stressoren Arbeitsmenge, Rollenstress, Klientenverhalten/-charakteristika und die personenbezogenen Faktoren Neurotizismus und Pflichtbewusstsein auf. Bei den Ressourcen gilt dies für die Kontrolle, Rollenklarheit, moderate Arbeitsmenge, Wertschätzung, Einbindung/Beteiligung und die personenbezogenen Ressourcen Werteübereinstimmung und Achtsamkeit.

Die gesichteten zehn Studien vermitteln einen ersten Eindruck über Faktoren, die mit der psychischen Gesundheit der Beschäftigten im untersuchten Berufsfeld im Zusammenhang stehen.

Praktische Relevanz Die Zahl der Beschäftigten in den Einrichtungen der Kinder- und Jugendhilfe steigt seit Jahren. Rund $21 \%$ der krankheitsbedingten Abwesenheitstage in der Berufsgruppe der Sozialarbeiter*innen sind auf psychische Erkrankungen zurückzuführen. Damit ist das Sozial- und Gesundheitswesen im Vergleich zu anderen Branchen besonders stark von psychischen und Verhaltens-Störungen betroffen.

Schlüsselwörter Sozialarbeiter*innen · Psychische Gesundheit · Belastungsfaktoren · Ressourcen · Stressoren

\section{Ambulatory Youth care: A systematic literature review on the Working and Health Situation}

\section{Abstract}

The diverse field of ambulatory social youth care and child protection is characterized by a number of psychological strains impacting the paedagogical professionals.

Julia C. Lengen, M.Sc.

j.lengen@uke.de

1 Zentralinstitut für Arbeitsmedizin und Maritime Medizin (ZfAM), Universitätsklinikum Hamburg Eppendorf (UKE), Seewartenstr. 10, Haus 1, 20459 Hamburg, Deutschland

2 Berufsgenossenschaft für Gesundheitsdienst und Wohlfahrtpflege, Pappelallee 33/35/37, 22089 Hamburg, Deutschland 
A systematic literature research was conducted in the time span 2000-2018 in the data bases Medline, PsyInfo und Psyndex using the PEO-Scheme to gain an overview of the working and health conditions of the target group. Relevant correlations were investigated. The involved articles were critically appraised with the checklists STROBE, PRISMA und CASP.

A variety of stressors and resources, mainly in the areas work content, work organisation and social relations are related to the social workers mental health. The strongest correlations have been found for the stressors work pressure/amount of work, role stress, clients behavior/characteristics and the person related factors neuroticism and conscientiousness. The resources control, role clarity, moderate workload, regard, involvement and the individual factors mindfulness and value congruence have an impact.

The ten reviewed studies provide a first impression of the relevant stressors and resources correlating with the mental health of the professionals in the researched occupational field.

Practical Relevance Concurrently, the number of social workers in institutions of child and youth welfare increased during the last years. About $21 \%$ of the sick leave days can be traced back to mental disorders. Thus, the social and health services are particularly concerned by mental and behavioral disorders.

Keywords Social worker $\cdot$ Mental health $\cdot$ Job demands $\cdot$ Ressources $\cdot$ Stressors

\section{Hintergrund}

\subsection{Soziale Arbeit}

Beschäftigte in der Sozialen Arbeit gehören neben den Gesundheits- und Lehrerberufen zu den Professionen, die überdurchschnittlich von beruflichem Stress betroffen sind (Grebner et al. 2010; Hussein 2018). Sozialpädagogische Berufe wie die ambulante Jugendhilfe sind durch Dienstleistungsarbeit charakterisiert (Glaser und Seubert 2014; Oechler 2009). Ihre Arbeit ist durch den persönlichen Kontakt, Beziehungsarbeit mit Klient*innen und einer hohen emotionalen Belastung im Sinne stetigen Erlebens des psychischen und physischen Leids der Klient*innen gekennzeichnet. Problematisch kann sich auch die duale Rolle von Kontrolle und Hilfe auswirken (Baldschun 2019; Hasenfeld 2010). Das Handeln der Sozialarbeiter*innen muss hochindividualisiert auf die oft widersprüchlichen Bedarfe der Klient*innen angepasst sein, wobei teilweise eine hohe Verantwortung für das Kindeswohl übernommen wird (Winkens 2016). Im branchenübergreifenden Vergleich treten einige Charakteristika des Berufes hervor: In den Erziehungs- und Sozialberufen werden besonders viele Arbeiten gleichzeitig ausgeführt, die Beschäftigten werden häufig mit neuen Aufgaben konfrontiert und es kommt im Vergleich zu anderen Berufen überdurchschnittlich oft zum Pausenausfall und Vereinbarkeitsproblemen. Mit 20\% sind die Sozial- und Erziehungsberufe die Berufsgruppe mit den dritthäufigsten Angaben ,an der Grenze der Leistungsfähigkeit“ zu arbeiten (Lohmann-Haislah 2012). Die Beschäftigten sind zudem von der Sorge vor körperlicher und psychischer Gewalt am Arbeitsplatz betroffen (Brodersen und Lück 2017; Steinlin et al. 2016). Als Ressourcen weisen die Sozial- und Erziehungsberufe einen vergleichsweise stark ausgeprägten Handlungsspielraum auf und fühlen sich am Arbeitsplatz überdurchschnittlich häufig als Teil einer Gemeinschaft (Lohmann-Haislah 2012).

In der Gesellschaft übernehmen die Beschäftigten in der Soziale Arbeit eine wichtige Aufgabe, indem sie Veränderungen und soziale Entwicklungen begleiten und den sozialen Zusammenhalt, die Autonomie und Selbstbestimmung von Menschen stärken. Ihr Ziel ist die Verbesserung des Wohlergehens der Menschen in schwierigen Lebenslagen (DBSH 2016; Scheu und Autrata 2018). Die Inanspruchnahme sozialer Dienstleistungen steigt in Deutschland seit Jahren und somit auch die Zahl der Beschäftigten in den Einrichtungen der Kinder- und Jugendhilfe (Winkens 2016). Insgesamt sind über 260.000 Menschen in der Kinder- und Jugendhilfe (ohne Tageseinrichtungen für Kinder) beschäftigt. Ein Großteil davon übt die pädagogische Tätigkeit ambulant aus (Bundesministerium für Familie 2014).

\subsection{Gesundheitssituation von Sozialarbeiter*innen}

Mehrere Übersichtsarbeiten weisen darauf hin, dass die Beschäftigten in der Sozialen Arbeit einem hohen Stresslevel ausgesetzt sind, welches sich negativ auf die psychische Gesundheit auswirken kann (Lloyd et al. 2002; Ben-Zur und Michael 2007). In verschiedenen internationalen Studien zeigen sich die langfristig negativen Beanspruchungsfolgen der Berufsgruppe, bspw. in Burnout, Sekundärtraumatisierung (McFadden et al. 2015) somatoformen Beschwerden (Poulsen 2014), reduzierter Leistungsfähigkeit (Baldschun 2019), krankheitsbedingter Abwesenheit, der Absicht, den Beruf aufzugeben sowie psychischen Erkrankungen wie Depressionen und Angstzuständen (Wooten et al. 2011). Laut dem Stressreport 2012 treten bei Beschäftigen in Sozial- und Erziehungsberufen überdurchschnittlich oft psychovegetative Beschwerden sowie körperliche und emotionale Erschöpfung auf (Lohmann-Haislah 2012). Wirth et al. (2019) haben aggregierte Routinedaten von vier Kran- 
kenkassen in Deutschland zum Krankenstand von 195.100 Sozialarbeiter*innen und Daten zu Unfallansprüchen von 3037 versicherten Sozialarbeiter*innen der Deutschen Gesetzlichen Unfallversicherung (DGUV) analysiert. Die Auswertung hat ergeben, dass psychische Erkrankungen in dieser Beschäftigtengruppe $21 \%$ der krankheitsbedingten Abwesenheitstage ausmachen. In der Allgemeinbevölkerung hat diese Erkrankungsgruppe laut dem SUGA-Report einen Anteil von 12,6\% (bei Männern 9,9\%; bei Frauen 15,9\%). In diesem Kontext ist zu berücksichtigten, dass rund $72 \%$ des Personals in Einrichtungen der Kinder- und Jugendhilfe weiblich sind (Statistisches Bundesamt 2018). Ein Bericht der Techniker Krankenkasse, der Arbeitsunfähigkeiten von Menschen in Sozial- und Erziehungsberufen aus dem Jahr 2019 nach Geschlecht ausweist, zeigt, dass Frauen mit 19,0 Arbeitsunfähigkeitstagen je Versicherungsjahr im Vergleich zu Männern mit 12,1 Tagen allgemein mehr Arbeitsunfähigkeitszeiten aufwiesen. Ebenso zeigt der Bericht, dass bei Frauen branchenunabhängig in den Jahren 2018 und 2019 häufiger psychische und Verhaltensstörungen diagnostiziert wurden (Grobe und Bessel 2020). Dass die Branche Gesundheit und Sozialwesen beispielsweise bei den Beanspruchungs- und Stressfolgen durch die Arbeit im Branchenvergleich laut dem Stress-Report an der Spitze stehen, führen die Autor*innen teilweise auch auf die Geschlechterverteilung zurück (Lohmann-Haislah 2012). Folglich steht das Gesundheits- und Sozialwesen auch bei der Betrachtung der Arbeitsunfähigkeitstage in Folge von psychischen und Verhaltens-Störungen pro Jahr an der Spitze der 24 betrachteten Branchen. Die Dauer der Arbeitsunfähigkeiten ist überdurchschnittlich lang (BAuA 2018). Trotz der Vielzahl an Beanspruchungen ist die Arbeitszufriedenheit der Beschäftigten in der Sozialen Arbeit vergleichsweise gut (Beckmann et al. 2009; Rose 2003).

\subsection{Zusammenhang zwischen der Arbeits- und Gesundheitssituation}

Steht die gesundheitliche Situation der Beschäftigtengruppe in der Sozialen Arbeit in Beziehung mit ihren Arbeitsbedingungen? Grundsätzlich haben Arbeit und Gesundheit eine wechselseitige Beziehung. Die Gesundheit wirkt sich auf die Arbeitsfähigkeit einer Person aus und arbeitsbezogene Stressoren und Ressourcen beeinflussen die Gesundheit (Gusy 2017). Der Mensch mit seinen individuellen Voraussetzungen ist eine wichtige Variable, wenn es um das gesamte Themengebiet „Belastung, Beanspruchung, psychische Gesundheit" geht (Paridon und Mühlbach 2016). So kann es aufgrund einer Erkrankung (z. B. Burnout oder Depression) ebenfalls zu einer negativeren Wahrnehmung der Arbeitssituation kommen. Arbeitsbedingungen und Beschäftigte stehen in permanenter Wechselwirkung. Um dem potenziellen Bias negativ empfundener Arbeitssituationen aufgrund des psychischen Zustands vorzubeugen, werden in Studien häufig objektive Messungen der Jobcharakteristika herangezogen (Lesener et al. 2019). Grundsätzlich zeigt die Studienlage, dass eine Vielzahl an Belastungsfaktoren in einem Zusammenhang mit der psychischen Gesundheit steht. Einzelne Faktoren zeichnen sich durch konsistente Zusammenhänge, zumeist in kleiner oder mittlerer Höhe, zur psychischen Gesundheit aus (Rothe et al. 2017). Neben psychischen Folgen (Rau et al. 2010) können auch somatische Erkrankungen auf arbeitsbedingte psychische Belastungsfaktoren zurückzuführen sein (Backe et al. 2012; da Costa und Vieira 2010). Neben den grundsätzlich gesundheitsförderlichen Auswirkungen von Arbeit gibt es diverse gesundheitsgefährdende Faktoren im Arbeitskontext (GDA 2017; Kramer et al. 2015; Rau and Henkel 2013; Schwartz 2007).

Die Arbeitsbedingungen in der sozialen Arbeit wurden bislang kaum systematisch untersucht und einzelne Studien sind nur schwer vergleichbar. Zudem ist die Anzahl an Studien zu gesundheitsförderlichen und -beeinträchtigenden Arbeitsbedingungen in der Sozialen Arbeit vergleichsweise gering. Für die Zielgruppe der Sozialarbeiter*innen in der Geflüchteten- und Wohnungslosenhilfe fanden Mette et al. (2020) heraus, dass die quantitativen und die emotionalen Anforderungen des Berufs als Stressoren im $\mathrm{Zu}-$ sammenhang mit Burnout stehen $(r=0,38$ und $r=0,44)$. Die Sinnhaftigkeit der Arbeit $(r=-0,24)$, soziale Unterstützung $(\mathrm{r}=-0,22)$ und Resilienz $(\mathrm{r}=-0,55)$ wirken als Ressourcen (Mette et al. 2020).

Neben vielen anderen Sozialarbeitergruppen ist die Arbeit in der ambulanten Kinder- und Jugendhilfe der Teil der Sozialen Arbeit, in dem insbesondere mit Kindern, Jugendlichen und Familien in deren Lebenswelt gearbeitet wird. Eine finnische Forschungsgruppe um Baldschun (2019) gab einen Hinweis auf eine negativere Ausprägung der psychischen Gesundheit bei dem im Kinder- und Jugendschutz tätigen pädagogischen Fachpersonal gegenüber ihren übrigen Kolleg*innen in der sozialen Arbeit mit Erwachsenen. Beim Vergleich beider Gruppen waren der Burnout-Score und der sekundärtraumatische Stress in der Gruppe der Sozialarbeiter*innen im Kinderschutz gegenüber der Vergleichsgruppe signifikant leicht erhöht. Bei der Arbeitszufriedenheit und dem selbst eingeschätzten generellen Gesundheitszustand zeigten sich keine signifikanten Unterschiede (Baldschun 2019; Hussein 2018). Das vielfältige Tätigkeitsfeld der Jugendhilfe im ambulanten Bereich ist von diversen - unter anderem psychischen - Einflussfaktoren geprägt, die sich auf die Gesundheit der pädagogischen Fachkräfte auswirken können (Bonde 2008).

Auch Barford und Whelton beschreiben die Arbeit in der ambulanten Kinder- und Jugendhilfe als einen anspruchsvollen Beruf, der mit emotional belastenden Situationen verbunden ist (Barford und Whelton 2010). Dauerhaft hohe 
Stresslevel - etwa durch die in Abschn. 1.1 beschriebenen Arbeitsanforderungen - können sowohl die Arbeitsqualität einschränken (Skirrow und Hatton 2007), als auch die psychische Gesundheit gefährden (Kim et al. 2011; Johnson et al. 2018; Nieuwenhuijsen et al. 2010).

Trotz der hohen gesellschaftlichen Relevanz der Tätigkeit und der hohen Beschäftigtenzahl ist das Belastungserleben der Zielgruppe in der ambulanten Jugendhilfe wenig beforscht (Allroggen et al. 2017; Nüsken 2020). Die Kombination aus

- hohen Arbeitsanforderungen,

- einer vergleichsweise schlechten psychischen Gesundheitssituation der Beschäftigten im Sozialwesen,

- die Hinweise auf bestehende Zusammenhänge beider (Baldschun 2019)

- sowie eine bisher lückenhafte arbeitspsychologische Forschungslage für die Zielgruppe der ambulanten Jugendhilfe (Allroggen et al. 2017)

machen Forschung in diesem Feld erforderlich.

\subsection{Fragestellung}

Aufgrund der aufgezeigten Forschungslücke wird in diesem Review der Frage nachgegangen, welche Stressoren und Ressourcen einen Einfluss auf die psychische Gesundheit der Beschäftigten in der ambulanten Jugendhilfe ausüben. Ziel ist es, den aktuellen Stand der Literatur hinsichtlich der relevanten verhältnis- und personenbezogenen Faktoren für die psychische Gesundheit der Zielgruppe zu identifizieren. Diese bieten potenzielle Ansatzpunkte für weitere arbeitswissenschaftliche Forschung zum Zusammenhang zwischen Arbeitsbedingungen und psychischer Gesundheit der Beschäftigten in der ambulanten Jugendhilfe und eine Verbesserung der gesundheitlichen Situation der Beschäftigtengruppe.

\section{Theoretischer Hintergrund}

Die Gemeinsame Deutsche Arbeitsschutzstrategie (GDA) hat im Arbeitsfeld ,psychische Gesundheit“ den Kenntnisstand aus der arbeitswissenschaftlichen Forschung gebündelt. In diversen Scoping-Studien wurde das verfügbare Wissen zur psychischen Gesundheit von der Bundesanstalt für Arbeitsschutz und Arbeitsmedizin (BAuA) aufbereitet (Rothe et al. 2017). Es wurden relevante Arbeitsbedingungsfaktoren sowie umfassende gesundheitliche Outcomes dokumentiert und die Befunde bewertet. Die Scoping-Studien zeigen generell, dass sich die Arbeitsfaktoren nach ihrer Wirkung in Stressoren und Ressourcen klassifizieren lassen. Unter Einbeziehung von Akteuren aus der Praxis verständigten sich die wissenschaftlichen Ex- pert*innen auf eine Kategorisierung der psychischen Gesundheit in relevante Arbeitsmerkmale der folgenden fünf Bereiche:

- Arbeitsinhalt

- Arbeitsorganisation

- Soziale Beziehungen

- Arbeitsumgebung (Schütte und Windel 2017)

- neue Arbeitsformen (Beck et al. 2017)

Diesen Bereichen lassen sich bedeutende Faktoren für eine menschengerechte Arbeitsgestaltung zuordnen (Rothe et al. 2017), sodass die Merkmalsbereiche als Kategoriensystem für die vorliegende Übersichtsarbeit genutzt werden können.

\section{Methodik}

\subsection{Definition der Zielgruppe}

Die gewählte Zielgruppe der ambulanten Jugendhilfe ist sehr heterogen. Sie umfasst ein breites Arbeitsfeld und ein umfangreiches Tätigkeitsprofil. Es wird an verschiedenen Arbeitsorten wie dem Büro, in sozialen Einrichtungen, in Privatwohnungen der Klient*innen oder an anderen Treffpunkten gearbeitet. Alle Fachkräfte eint die Arbeit mit Menschen in prekären Situationen (Winkens 2016).

Die Leistungen der Kinder- und Jugendhilfe werden durch öffentliche Jugendämter, Träger der freien Jugendhilfe, kirchliche Träger und privatwirtschaftliche Unternehmen erbracht (Bundesministerium für Familie 2014).

Die Zielgruppe des Literaturreviews sind ambulant arbeitende, hauptamtlich Beschäftigte in der Erziehungsberatung, der sozialpädagogischen Familienhilfe, in den ambulanten Hilfen zur Erziehung oder der offenen Jugendarbeit. Ausgeschlossen wurden Personen, die in Behörden, also etwa im Allgemeinen Sozialen Dienst oder in Jugendämtern tätig sind, da ihr Tätigkeitsspektrum und die damit verbundenen Stressoren und Ressourcen sowie die Rahmenbedingungen des Arbeitgebers sich unterscheiden. Die Tätigkeiten eines Allgemeinen Sozialen Dienstes sind breiter gefächert, als die innerhalb der erzieherischen Hilfe und enthalten höhere Anteile an Verwaltungstätigkeiten (Nüsken 2020).

\subsection{Literatursuche}

Die Forschungsfrage wird mit Hilfe des PEO-Schemas (Bettany-Saltikov 2012) folgendermaßen operationalisiert:

- Population: Beschäftigte mit sozialpädagogischen Tätigkeiten in den unterschiedlichen Bereich der ambulanten Jugendhilfe 


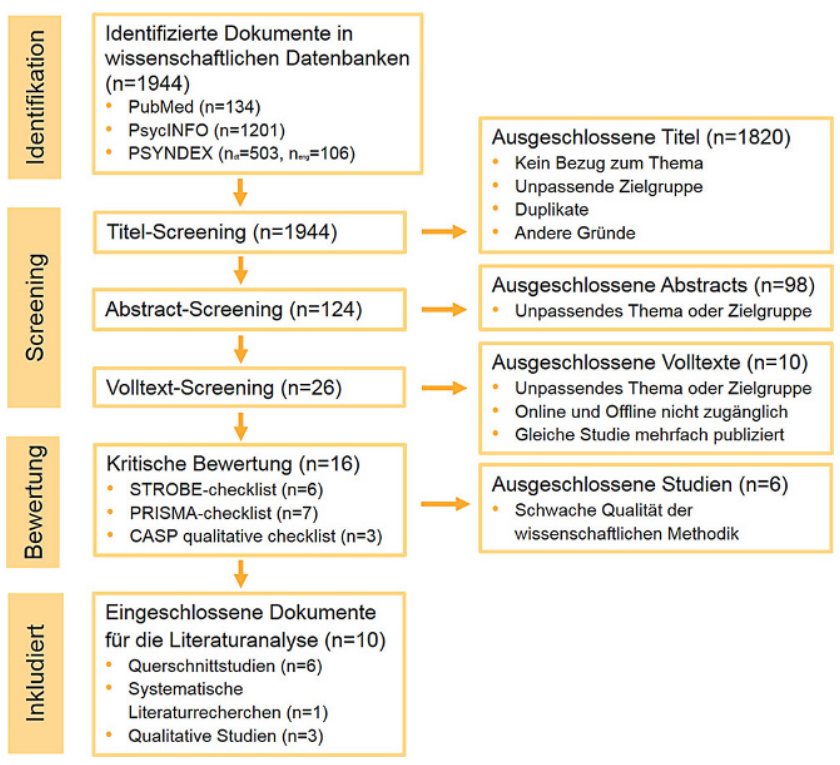

Abb. 1 Flussdiagramm der Studienauswahl

Fig. 1 Flow chart of study selection

- Exposition: Arbeitsbedingungen, psychische Belastungsfaktoren (z. B. Stressoren, Ressourcen)

- Outcome: psychischer Gesundheitsstatus, Prävalenz von psychischen Beschwerden und Erkrankungen, Burnoutund Stress-Symptomatik, Wohlbefinden

Die Suchbegriffe (s. Anhang, Anlage 1) wurden in Absprache mit einer Gruppe von wissenschaftlichen Expertinnen ausgewählt. Ein Rückkopplungsprozess der englischen Begriffe fand mit einer Fremdsprachenkorrespondentin statt.

Die Literatursuche wurde im Zeitraum vom 11.12.2018 bis 03.01.2019 durchgeführt und bezog sich auf die Datenbanken PsychINFO, Medline und PSYNDEX.. Die Suche in diesen medizinischen und arbeitspsychologischen Datensammlungen wurde aufgrund des thematischen Hintergrunds als zielführend erachtet. Ohne weitere Filter wurde englisch- und deutschsprachige Literatur im Veröffentlichungszeitraum von 2000 bis 2018 gesucht.

Von den ausgegebenen Artikeln wurden der Titel, das Abstract und anschließend der Volltext gelesen und jeweils themen- oder zielgruppenfremde Artikel und unzugängliche Volltexte ausgeschlossen (s. Abb. 1). Es ergaben sich insgesamt 16 Studien mit qualitativen und quantitativen Studiendesigns und systematische Literaturreviews aus dem Screeningprozess.

Mit Hilfe der Checklisten PRISMA - (für Übersichtsarbeiten), STROBE - (für quantitative Studien) und CASP (für qualitative Studien) (Moher et al. 2009; Vandenbroucke et al. 2007; CASP 2018) wurden die inhaltlich passenden Artikel von zwei Reviewerinnen auf ihre Qualität überprüft. Die Checklistenkriterien wurden in einer Excel-
Tabelle von beiden Reviewerinnen unabhängig voneinander auf die 16 vorliegenden Artikel angewandt und anschließend abgeglichen. Das Cohens Kappa von initial 0,75 deutet auf eine gute Inter-rater-Reliabilität hin (Jacobs 2008; Krüger et al. 2014). Bei einer gegenseitigen Erläuterung der Hintergründe und einer Beratung über unterschiedliche Ergebnisse konnten Einigungen erzielt werden. 10 der 16 bewerteten Studien haben die Qualitätsprüfung (mit Erfüllungsgraden von 50 bis $90 \%$ ) bestanden und wurden für das Review ausgewertet. Im Prozess der kritischen Bewertung wurden sechs Studien aufgrund ihrer schwachen wissenschaftlichen Methodik (z. B. unsystematische Suche bei Übersichtsartikeln) ausgeschlossen.

Die Daten der ausgewählten Artikel wurden extrahiert, tabellarisch für jede einzelne Studie zusammengefasst und die Studienmethodik sowie die erkannten Zusammenhänge zwischen den erhobenen psychischen Belastungsfaktoren und den gesundheitlichen Outcomes detailliert aufgeführt (s. Anlage 2). Die Ergebnisse wurden analysiert und strukturiert, indem die Prädiktoren sortiert und ihre Zusammenhänge mit den Outcomes grafisch dargestellt wurden. Eine gute Übersicht gewährt die Strukturierung nach den in Kap. 2 beschriebenen Merkmalen der Gemeinsamen Deutschen Arbeitsschutzstrategie (Beck et al. 2017) (s. Abb. 3).

\section{Ergebnisse}

Mittels einer systematischen Literaturrecherche konnten zehn Studien identifiziert werden, die die Arbeits- und Gesundheitssituation von Beschäftigten in der ambulanten Jugendhilfe untersuchen und den Qualitätsanforderungen entsprechen. Einbezogen wurden drei qualitative Studien, sechs Querschnittstudien und ein systematisches Review. Der Großteil der Studien untersuchte innerhalb der letzten vier Jahre die Arbeitssituation in Europa (s. Tab. 1).

Im Folgenden werden die Rechercheergebnisse zum Zusammenhang psychischer Belastungsfaktoren (Ressourcen oder Stressoren) mit den Outcomes dargestellt. Da eine grafische Darstellung einzelner Zusammenhänge zu unübersichtlich erscheint, wurden die Daten grafisch in der Abb. 3 gruppiert zusammengestellt. Detaillierte Informationen zu einzelnen erhobenen Zusammenhängen können dem Anhang 2 entnommen werden.

Die Legende (s. Abb. 2) erläutert die Darstellungsweise in der Ergebnistabelle (s. Abb. 3). Die hochgestellten Zahlen und Buchstaben geben die Primärquellen und die Studienart an. Anhand dessen ist ersichtlich, welche $\mathrm{Zu}$ sammenhänge zwischen Prädiktoren und Outcomes in den jeweiligen Studien untersucht wurden. Signifikante Zusammenhänge sind in schwarz dargestellt, nicht signifikante in grau. Starke Zusammenhänge sind in fett dargestellt, kon- 
Tab. 1 Überblick der Studiencharakteristika $(n=10)$

Table 1 Overview of the study characteristics $(n=10)$

\begin{tabular}{lll}
\hline Charakeristika & $n$ & $\%$ \\
\hline Studiendesign & & \\
Qualitative Studien & 3 & 30 \\
Quantitative Querschnittstudien & 6 & 60 \\
Review/Literaturrecherche & 1 & 10 \\
Ort der Studie & & \\
Großbritannien & 3 & 30 \\
Kanada & 1 & 10 \\
Deutschland & 3 & 30 \\
Israel & 1 & 10 \\
Australien & 1 & 10 \\
Global & 1 & 10 \\
Jahr der Publikation & & \\
2015-2018 & 7 & 70 \\
2010-2014 & 2 & 20 \\
2005-2009 & 1 & 10 \\
$<2005$ & 0 & 0 \\
\hline
\end{tabular}

trovers diskutierbare mit verschiedenen Ergebnissen in den unterschiedlichen Studien in kursiv.

Als gesundheitlicher Outcome wurden in mehreren Studien die Burnout-Komponenten emotionale Erschöpfung, Depersonalisierung und persönliche Leistung gemessen (Maslach und Jackson 1981; Biggart et al. 2017; Poulsen 2014; McFadden et al. 2015). Des Weiteren wurden sekundärtraumatischer Stress (Bride et al. 2004), der allgemeine Gesundheitszustand (Goldberg 1972), und psychologischer Disstress (Harker et al. 2016) erhoben. Auch das Auftreten psychischer Erkrankungen (Antonopoulou et al. 2017), Selbstvertrauen (Biggart et al. 2017; McFadden et al. 2015), Fluktuationen bzw. die Intention zum Jobwechsel (McFadden et al. 2015) wurden untersucht. Qualitativ beschriebene gesundheitliche Outcomes waren beispielsweise das Wohlbefinden, die psychische Gesundheit (Biggart et al. 2017), Panikattacken, Schlafbeschwerden, somatoforme Beschwerden an Kopf, Magen und Rücken, Erschöpfung

\begin{tabular}{|c|c|}
\hline \multicolumn{2}{|c|}{ Quellenangaben unterschieden nach Studienart } \\
\hline $\begin{array}{l}\text { Quantitative } \\
\text { Studien }\end{array}$ & \begin{tabular}{|l|}
${ }^{1}$ Harker, Pidgeon, Klaassen, and King (2016) \\
${ }^{2}$ Dagan et al. (2016) \\
${ }^{3}$ McFadden et al. (2018) \\
${ }^{4}$ Antonopoulou, Killian \&Forrester (2017) \\
${ }^{5}$ Barford and Whelton (2010) \\
${ }^{6}$ Beckmann et al. (2009) \\
\end{tabular} \\
\hline $\begin{array}{l}\text { qualitative } \\
\text { Studien }\end{array}$ & $\begin{array}{l}{ }^{a} \text { Winkens (2016) } \\
{ }^{b} \text { Poulsen (2014) } \\
{ }^{c} \text { Biggart, Ward, Cook \&Schofield (2017) }\end{array}$ \\
\hline Review & ${ }^{D}$ McFadden et al. (2015) \\
\hline \multicolumn{2}{|c|}{ Visuelle Darstellung der Zusammenhänge } \\
\hline schwarz & signifikant \\
\hline grau & nicht signifikant \\
\hline fettgedruckt & $r$ bzw. $\beta>0,25$ \\
\hline normalgedruckt & $r$ bzw. $\beta<0,25$; oder qualitativ \\
\hline kursiv gedruckt & $\begin{array}{l}\text { kontroverse Ergebnisse in den Studien hinsichtlich Signifikanz (und } \\
\text { Effektstärke) }\end{array}$ \\
\hline
\end{tabular}

Abb. 2 Legende für die Ergebnistabelle Abb. 3

Fig. 2 Legend for results Fig. 3
(Poulsen 2014) und Resilienz (McFadden et al. 2015; Winkens 2016).

In der Kategorie Arbeitsinhalt zeigten sich die stärksten signifikanten Zusammenhänge für die oben genannten Outcomes bei den Stressoren: Rollenstress (Dagan et al. 2016; Poulsen 2014) und Klientenverhalten und -charakteristika. Auch mit dem Beruf verbundene emotionale Inanspruchnahme (Dagan et al. 2016; Winkens 2016; Poulsen 2014), fehlende Vorhersehbarkeit und hohe Verantwortung (Poulsen 2014) stellen relevante Stressoren dar. Als wichtigste Ressourcen wirken Kontrolle über die eigene Arbeit, klar kommunizierte Werte/Rollenklarheit (Antonopoulou et al. 2017; Barford und Whelton 2010; Winkens 2016). Des Weiteren wirken sich offenbar Weiterbildungsmöglichkeiten (Poulsen 2014; Antonopoulou et al. 2017; McFadden et al. 2015) und Rückmeldung aus der Tätigkeit (Biggart et al. 2017; Beckmann et al. 2009) sowie Ganzheitlichkeit (Beckmann et al. 2009) positiv auf die psychischen Gesundheitsoutcomes der untersuchten Berufsgruppe aus. Die gemessenen Effekte von Jobautonomie und die Bedeutsamkeit der Aufgabe sind umstritten oder nicht signifikant (Antonopoulou et al. 2017; Barford und Whelton 2010; Beckmann et al. 2009).

Im Bereich Arbeitsorganisation zeigen eine große Arbeitsmenge bzw. Arbeitsdruck (McFadden et al. 2018, 2015; Barford und Whelton 2010; Poulsen 2014; Biggart et al. 2017) den stärksten Zusammenhang mit den gesundheitlichen Outcomes Burnout, Stress, psychosomatischen Beschwerden, Selbstvertrauen und dem Wohlbefinden. Auch mangelnde personelle und finanzielle Mittel bzw. eine vorliegende Diskrepanz zum Bedarf der Klient*innen, Kontrolle und Bürokratie von Politik und Behörden sind relevante Stressoren für die psychische Gesundheit. McFadden et al. beschreiben einen vergleichsweise starken signifikanten Zusammenhang zwischen einer moderaten Arbeitsmenge und einer niedrigeren Zahl von Burnouterkrankungen (McFadden et al. 2018). Auch die Ressourcen Supervisionen (Winkens 2016; Dagan et al. 2016; Poulsen 2014; Biggart et al. 2017; McFadden et al. 2015), organisatorische Unterstützung (Antonopoulou et al. 2017; McFadden et al. 2015) und Zeit für Austausch mit Kolleg*innen (Winkens 2016; Poulsen 2014) werden als gesundheitsrelevante Ressourcen erachtet. Fairness und Gerechtigkeit erweisen sich hingegen nicht eindeutig als Ressource gegen Burnout (McFadden et al. 2018).

Bei den sozialen Beziehungen sind die Zusammenhänge bzw. Korrelationen zum gesundheitlichen Outcome nierdriger als bei der Arbeitsorganisation und den Arbeitsinhalten. Jedoch wirken sich offenbar Konflikte mit Führungskräften als Stressor auf die psychische Gesundheit aus (Poulsen 2014; Biggart et al. 2017). In qualitativen Studien wurden auch überarbeitete Kolleg*innen (Poulsen 2014), eine offenkundig effizienzorientierte Führung (Biggart et al. 


\begin{tabular}{|c|c|c|c|c|c|c|c|}
\hline \multicolumn{5}{|c|}{ Arbeitsmerkmale } & \multirow[b]{2}{*}{$\begin{array}{l}\text { Personenbezogene } \\
\text { Faktoren }\end{array}$} & & \multirow[b]{2}{*}{ Gesundheitlicher Outcome } \\
\hline Arbeitsinhalt & Arbeitsorganisation & $\begin{array}{l}\text { Soziale } \\
\text { Beziehungen }\end{array}$ & $\begin{array}{l}\text { Arbeits- } \\
\text { umgebung }\end{array}$ & $\begin{array}{l}\text { Neue Arbeits- } \\
\text { formen }\end{array}$ & & & \\
\hline 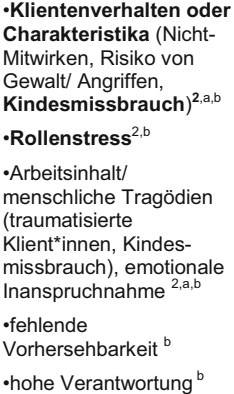 & $\begin{array}{l}\text {-Arbeitsdruck, zu } \\
\text { hohe Arbeitsmenge } \\
b, c, D \\
\text { •hohe Fluktuation }{ }^{\mathrm{c}} \\
\text { (hoher Einarbeitungsaufwand) } \\
\text { •nicht ausreichend } \\
\text { personelle und } \\
\text { finanzielle Ressourcen } \\
\text { (Diskrepanz zum } \\
\text { Bedarf der Klienten - } \\
\text { Zeitdruck) } \\
\text { •Kontro } \\
\text { und der Behö Pörditik } \\
\text { Bürokratie }\end{array}$ & $\begin{array}{l}\text { •Konflikte mit } \\
\text { Führungskräften } \\
\text { oder Super- } \\
\text { visor*innen }^{b, c} \\
\text {-überarbeitete } \\
\text { Kolleg*innen }^{\mathrm{b}} \\
\text {-offen } \\
\text { effizienzorientierte }^{*} \\
\text { Führung }^{\mathrm{c}} \\
\text {-geringe } \\
\text { Bezahlung, } \\
\text { mangelnde } \\
\text { Wertschätzung }\end{array}$ & $\begin{array}{l}\text {-Arbeits- } \\
\text { umgebung }\end{array}$ & •Entgrenzung ${ }^{\mathrm{b}}$ & $\begin{array}{l}\cdot{ }^{\cdot} \text { Neurotizismus }{ }^{5} \\
\cdot \text { Pflichtbewusstsein }{ }^{5} \\
\text { •eigene traumatische } \\
\text { Erfahrungen }{ }^{2, D} \\
\text {-schwach ausgeprägte } \\
\text { Abgrenzungsfähigkeit }{ }^{\text {b }}\end{array}$ & 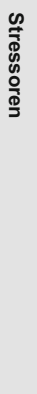 & 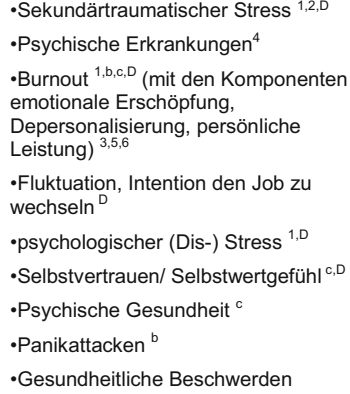 \\
\hline $\begin{array}{l}\text { •Kontrolle }{ }^{3} \\
\text { •klarkommunizierte } \\
\text { Werte/ Rollenklarheit } \\
4,5, \mathrm{a} \\
\text { •hoch qualifiziertes } \\
\text { Personal, Weiter- } \\
\text { bildungsmöglichkeiten } \\
4, \mathrm{~b}, \mathrm{D}, \text { Jobperspektiven }^{4} \\
\text {-Rückmeldung aus der } \\
\text { Tätigkeit }{ }^{6, \mathrm{c}} \\
\text {-Ganzheitlichkeit }{ }^{6} \\
\text { - Jobautonomie, Hand- } \\
\text { lungsspielraum }{ }^{5,6, \mathrm{~b}} \\
\text { •bedeutsame Aufgabe, }^{\text {Aufgabenvielfalt }}{ }^{6}\end{array}$ & $\begin{array}{l}\text {-moderate } \\
\text { Arbeitsmenge }{ }^{3} \\
\text {-Supervision } 2, a, b, c, D \\
\text { •kleine Teams }{ }^{4} \\
\text {-organisatorische } \\
\text { Unterstützung und } \\
\text { Organisationskultur }{ }^{4, D} \\
\text {-Zeit für Austausch im } \\
\text { Kollegium }^{\mathrm{a}, \mathrm{b}, \mathrm{c}}\end{array}$ & $\begin{array}{l}\text { •Empfundene } \\
\text { Wertschätzung }^{3, \mathrm{c}, \mathrm{D}} \\
\text { •Einbindung/ } \\
\text { Beteiligung }^{5} \\
\text {-regelmäßiger } \\
\text { Austausch im } \\
\text { Kollegium }^{\mathrm{a}, \mathrm{c}, \mathrm{D}} \\
\text {-Kooperation im } \\
\text { Team }^{\mathrm{a}, \mathrm{c}} \\
\text { •empfundene } \\
\text { soziale Unter- } \\
\text { stützung }^{2,5, \mathrm{a}, \mathrm{b}, \mathrm{c}, \mathrm{D}} \\
\text {-Rückmeldung/ } \\
\text { Unterstützung der } \\
\text { Führungskraft }\end{array}$ & & & 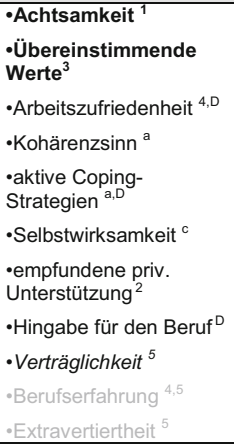 & 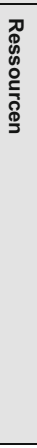 & $\begin{array}{l}\text { (Schlaf-störungen, } \\
\text { Rückenschmerzen, Probleme zu } \\
\text { entspannen, Müdigkeit, } \\
\text { Kopfschmerzen, } \\
\text { Magenbeschwerden, Erschöpfung) }^{\mathrm{b}} \\
\text { •Arbeitseffizienz }{ }^{\mathrm{c}} \\
\text { •Persönliche und organisationale } \\
\text { Resilienz }^{\mathrm{a}, \mathrm{D}} \\
\text {-Wohlbefinden }{ }^{\mathrm{c}} \text { (bei der Arbeit) }{ }^{3,4}\end{array}$ \\
\hline
\end{tabular}

Abb. 3 Ergebnisse der systematischen Literaturrecherche

Fig. 3 Results of the systematic literature search

2017) und in einem Review die geringe Bezahlung sowie mangelnde Wertschätzung (McFadden et al. 2015) als belastend beschrieben. Soziale Ressourcen, die starke Zusammenhänge zur psychischen Gesundheit aufweisen, sind die Einbindung/Beteiligung der Beschäftigten in die Organisation (McFadden et al. 2015; Barford und Whelton 2010) sowie empfundene Wertschätzung (McFadden et al. 2018; Biggart et al. 2017; McFadden et al. 2015). Geringere, jedoch ebenfalls signifikante Zusammenhänge, weisen regelmäßiger Austausch unter Kolleg*innen und Kooperation im Team (Winkens 2016; Biggart et al. 2017; McFadden et al. 2015) auf. Die Themen Feedback und Unterstützung der Führung und die empfundene soziale Unterstützung stellen nicht in allen Studien signifikante Ressourcen dar (Barford und Whelton 2010; Beckmann et al. 2009; Biggart et al. 2017; McFadden et al. 2015), sodass ihre Wirkung als Gesundheitsressource laut der Studienlange umstritten ist.

Bezüglich der Zusammenhänge zwischen Arbeitsumgebung (in den Studien operationalisiert als körperliche Arbeitsbedingungen bzw. körperlicher Komfort im Arbeitsumfeld) und der Gesundheit ist die Studienlage nicht eindeutig (Antonopoulou et al. 2017; Barford und Whelton 2010).

Bei Berücksichtigung der neuen Arbeitsformen wird Entgrenzung von Arbeit (von Poulsen beschrieben als die tätigkeitsbedingte Schwierigkeit, sich abzugrenzen, die eigenen Grenzen wahrzunehmen und zu ziehen) als Stressor wahrgenommen (Poulsen 2014). Dieser tätigkeitsbezogene Fak- tor steht im Zusammenhang mit dem personenbezogenen Faktor der mangelnden Abgrenzungsfähigkeit.

Die Untersuchung einiger personenbezogener Faktoren deutet darauf hin, dass stark ausgeprägter Neurotizismus und Pflichtbewusstsein (Barford und Whelton 2010) die deutlichsten signifikanten Zusammenhänge mit dem Gesundheitsoutcome Burnout aufweisen. Auch eigene traumatische Erfahrungen stellen einen personenbezogenen Risikofaktor für die psychische Gesundheit dar (Dagan et al. 2016; McFadden et al. 2015). Achtsamkeit (Harker et al. 2016) und übereinstimmende Werte von Arbeitnehmer*in und Organisation (McFadden et al. 2018) stellen deutliche Ressourcen mit vergleichsweise starken Zusammenhängen dar. Auch die Nutzung aktiver Coping-Strategien (Winkens 2016; McFadden et al. 2015), die Zufriedenheit mit der Arbeit (Antonopoulou et al. 2017; McFadden et al. 2015), ein ausgeprägter Kohärenzsinn (Winkens 2016) Selbstwirksamkeit (Biggart et al. 2017) und Hingabe für die Tätigkeit (McFadden et al. 2015) stellen der Literatur zufolge persönliche Ressourcen für die psychische Gesundheit der Berufsgruppe dar. Berufserfahrung und Extravertiertheit wiesen keine signifikanten Zusammenhänge zur psychischen Gesundheit auf (Antonopoulou et al. 2017; Barford und Whelton 2010). 


\section{Diskussion}

\subsection{Zusammenfassung}

Das systematische Literaturreview zeigt einige Zusammenhänge zwischen den untersuchten Arbeitsbedingungen in den Kategorien Arbeitsinhalt, -organisation, -umgebung, soziale Beziehungen und personenbezogene Faktoren und dem Outcome psychische Gesundheit auf. Es wurde ein Überblick zum wissenschaftlichen Erkenntnisstand für die Arbeitssituation in der ambulanten Jugendhilfe erarbeitet, auf dessen Basis Forschungsergebnisse und -lücken sichtbar werden.

Bisherigen Forschungsergebnissen zufolge stellen sowohl verhältnis- als auch personenbezogene Faktoren deutliche Stressquellen dar (Wooten et al. 2011). In der Literatur wurden für verschiedene Stressoren deutlich negative Zusammenhänge mit der psychischen Gesundheit der Zielgruppe dargestellt: Rollenstress, Klientenverhalten, große Arbeitsmengen sowie individuell stark ausgeprägter Neurotizismus und Pflichtbewusstsein. Als besonders relevante Ressourcen dienen der Beschäftigtengruppe in der ambulanten Jugendhilfe Kontrolle über die eigene Arbeit, Rollenklarheit, moderate Arbeitsmengen, Wertschätzung, Beteiligungsmöglichkeiten sowie die personenbezogenen Ressourcen übereinstimmende Werte und Achtsamkeit.

Besonders deutliche Zusammenhänge zwischen psychischen Belastungsfaktoren und gesundheitlichem Outcome ergeben sich folglich in der GDA-Kategorie Arbeitshalt. Dort weisen sowohl einige Stressoren als auch einige Ressourcen mittlere signifikante Zusammenhänge zu den Gesundheitsindikatoren auf. Dies deutet auf die besondere Relevanz der Stressoren und Ressourcen, die sich aus den Arbeitsinhalten ergeben, hin.

\subsection{Einordnung in die Forschungslandschaft}

Die hier zusammengefassten Forschungsergebnisse zu Beschäftigten in der ambulanten Jugendhilfe ähneln in weiten Teilen den Erkenntnissen der arbeitspsychologischen Forschung der gesamten Gruppe der Sozialarbeiter*innen (vgl. Abschn. 1.2 und 1.3) (Baldschun 2019; Lloyd et al. 2002; Mette et al. 2020).

Baldschuns Forschungserkenntnisse (2019) deuten auf eine leicht negativere Ausprägung der Outcomes Burnout und sekundärtraumatischer Stress bei Beschäftigten im Kinder- und Jugendschutz gegenüber dem Fachpersonal, das mit Erwachsenen tätig ist, hin. Möglicherweise kann dies im spezifischen Tätigkeitsspektrum der Arbeit mit Kindern und Jugendlichen begründet sein. Verglichen mit Sozialarbeiter*innen in anderen Bereichen gibt es eine hohe Verantwortung für das Kindeswohl, eine erhöhte Exposition gegenüber Kontexten mit Kindesmissbrauch (Dagan et al.
2016) sowie stärker ausgeprägte Rollenkonflikte, da sowohl Hilfe als auch Kontrolle gewährleistet werden müssen (Baldschun 2019; Hasenfeld 2010).

In Ergänzung zur ambulanten Jugendhilfe bilden in stationären Jugenhilfesettings Grenzverletzungen (Beschimpfungen, Drohungen und tätliche Angriffe) einen zusätzlichen Belastungsschwerpunkt. Vor diesem Hintergrund wurden Zusammenhänge zu psychischen und körperlichen Erschöpfungssymptomen, posttraumatischer Symptomatik als Folge von bedrohlichen Erlebnissen sowie Symptome von Sekundärtraumatisierung benannt. Zurückgeführt wird sie unter anderem auf das Befassen mit den lebensgeschichtlichen Belastungen der Kinder und Jugendlichen (Steinlin et al. 2016). Ein direkter Vergleich zwischen ambulanter und stationäre Jugendhilfe wird in keiner der vorliegenden Studien unternommen. Um zu verstehen, inwiefern sich die Arbeitsbelastungen der Beschäftigten in der ambulanten Jugendhilfe von anderen pädagogischen Fachkräften unterscheiden, böte sich die Erforschung der jugendhilfespezifischen Charakteristika sowie eine Untersuchung der spezifischen Belastungen des ambulanten Settings an. Auch ein Vergleich mit angrenzenden Berufsfeldern wie der stationären Jugendhilfe erscheint sinnvoll.

\subsection{Limitationen}

Die definierte Zielgruppe ambulante Jugendhilfe ist sehr heterogen und folglich sind es auch die jeweiligen Populationen der einbezogenen Studien. Die Stichproben bilden in mehreren Fällen nicht die Bandbreite der definierten Zielgruppe ab oder enthalten teilweise Personen außerhalb der Zielgruppe. So werden beispielsweise in einer Studie ausschließlich „Child protection workers" (McFadden et al. 2015) oder Fachkräfte aus der Sozialpädagogischen Familienhilfe (Beckmann et al. 2009) untersucht, die nur einen kleinen Teil der Zielgruppe ausmachen. In einer anderen Studie von Harker et al. (2016) wurden neben den passenden Berufsgruppen ,social work, counseling und youth and foster care" auch Psycholog*innen, die nicht der hier definierten Zielgruppe angehören, beforscht.

Aufgrund der schwachen Studienlage zu der definierten Zielgruppe wurden für die kritische Bewertung der Studienqualität mittels Checklisten (Moher et al. 2009, Vandenbroucke et al. 2007; CASP 2018) moderate Ansprüche mit Erfüllungsquoten von $\geq 50 \%$ gestellt. Durch den Einsatz von zwei Reviewerinnen wurde der Prozess objektiviert.

Trotz der geringen Studienanzahl erschwert die Vielzahl unterschiedlicher Arbeitsanforderungen und gesundheitlichen Outcomes die Auswertung. In der zusammenfassenden Schlussfolgerung können Informationen über spezifische Zusammenhänge nicht gänzlich abgebildet werden. Die verschiedenen Outcomes wurden weitgehend auf den 
größten gemeinsamen Nenner, die psychische Gesundheit, reduziert.

Limitierend für das Ableiten einer allgemeingültigen Schlussfolgerung ist die internationale Suche. Es bleibt unklar, inwieweit die Situation in den beforschten Ländern vergleichbar ist, da sich die Sozial- und Gesellschaftssysteme global stark unterscheiden. Auch kulturelle Unterschiede, die divergierenden Forschungsfragen und Herangehensweisen beeinflussen die Studienergebnisse. Ein auf Deutschland beschränktes Review gewährt jedoch aufgrund der wenigen Studien nicht ausreichend Aussagekraft.

Insgesamt konnte mit zehn Studien eine recht geringe Anzahl von Studien zum untersuchten Thema ausgewertet werden. Da die Untersuchung keine Längsschnittstudien ergab, können keine Kausalzusammenhänge abgeleitet werden.

\subsection{Implikationen für die Forschung}

Die Ergebnisse der Literaturrecherche deuten auf eine moderierende Wirkung einiger psychischer Belastungsfaktoren hin. In der qualitativen Studie von Biggart et al. (2017) wird die Unterstützung im Team für den Zusammenhang einiger psychischer Belastungsfaktoren (z.B. Bewältigen großer Mengen Arbeit) als moderierender Faktor zum Outcome psychische Gesundheit diskutiert. Poulsen (2014) beschrieb, dass eine hohe Arbeitsmenge und Zeitdruck dazu führen, dass andere Stressoren (wie z.B. die emotionale Inanspruchnahme und hohe qualitative Anforderungen) vom Individuum schlechter bewältigt werden können. Die in mehreren Studien beforschte Ressource soziale Beziehungen zeigte uneinheitliche Ergebnisse, sodass auch hier andere Moderator- oder Mediatoreffekte eine Rolle spielen könnten. In den Feldern „Feedback und Unterstützung der Führung“ und „empfundene soziale Unterstützung“ zeigten sich divergierende Ergebnisse. Eine Betrachtung der Operationalisierung und eine genauere Analyse einzelner Teilaspekte könnte weitere Erkenntnisse hinsichtlich der Relevanz sozialer Beziehungen für die Berufsgruppe offenbaren. Die hier beschriebenen Moderationseffekte bedürfen jedoch weiterer empirischer Belege.

Die geringe Anzahl und Qualität der Studien deuten darauf hin, dass der Zusammenhang von psychischen Belastungsfaktoren und psychischer Gesundheit bei dieser speziellen Berufsgruppe noch nicht ausreichend erforscht ist. Zudem liegt keine Studie vor, die die Zielgruppe in Deutschland umfassend untersucht hat und daraus praxistaugliche Handlungsempfehlungen ableiten konnte. Daraus ergibt sich ein weiterer Forschungsbedarf, sowohl in der Grundlagen- als auch in der Interventionsforschung.

\section{Fazit \& Ausblick}

Ziel des vorliegenden Reviews war die Darstellung eines Überblicks der Zusammenhänge zwischen den in der ambulanten Jugendhilfe bestehenden psychischen Belastungsfaktoren und der psychischen Gesundheit der dort Beschäftigten. Es wurden verschiedene gesundheitsrelevante arbeitsund personenbezogenen Faktoren der Fachkräfte herausgearbeitet. Wichtige im Betrieb veränderbare psychische Belastungsfaktoren befinden sich vor allem in den Arbeitshalten, der Arbeitsorganisation und den sozialen Beziehungen. Gerade im Bereich der sozialen Ressourcen sind die Forschungsergebnisse inkonsistent.

Nur wenige Studien haben die gesundheitsrelevanten psychischen Belastungsfaktoren und ihre Auswirkungen in der ambulanten Jugendhilfe beforscht. Mittels dieser Literatursichtung und der Analyse und Einordnung bisheriger Forschungsergebnisse konnte ein erster Einblick in die Arbeitssituation der betrachteten Fachkräftegruppe gewonnen werden. Das Literaturreview subsummiert die beforschten Faktoren, stellt ihre Zusammenhänge zur psychischen Gesundheit dar und zeigt Forschungsbedarfe auf. Diese Aufbereitung der gesundheitsrelevanten Themen für die besagte Zielgruppe kann weiterer arbeitswissenschaftlicher Forschung in diesem Feld als Grundlage dienen.

Förderung Die Studie wurde von der Berufsgenossenschaft für Gesundheitsdienst und Wohlfahrtpflege finanziert.

Funding Open Access funding enabled and organized by Projekt DEAL.

\section{Einhaltung ethischer Richtlinien}

Interessenkonflikt J. Lengen, M. Kersten und S. Gregersen geben an, dass kein Interessenkonflikt besteht.

Ethische Standards Alle beschriebenen Untersuchungen am Menschen oder an menschlichem Gewebe wurden mit Zustimmung der zuständigen Ethikkommission, im Einklang mit nationalem Recht sowie gemäß der Deklaration von Helsinki von 1975 (in der aktuellen, überarbeiteten Fassung) durchgeführt. Von allen beteiligten Patienten liegt eine Einverständniserklärung vor.

\section{Anhang}

\section{Anlage 1}

\section{Suchstrategie in Pubmed}

1. Social Work (population)

2. Working condition (exposition)

3. Health Outcome

4. 1 AND 2 AND 3

5. Limit 4 to Publication date from $2000 / 01 / 01$ 
Social Work "Social work"[Title/Abstract] OR "Social worker"[Title/Abstract] OR "educational counselling" [Title/ Abstract] OR "youth work"[Title/Abstract] OR "youth worker"[Title/Abstract] OR "youth care"[Title/Abstract] OR "youth service" [Title/Abstract] OR "outreach work" [Title/Abstract] OR "outreach worker"[Title/Abstract] OR "community work" [Title/Abstract] OR "community worker"[Title/Abstract] OR "drug prevention"[Title/Abstract] OR "family support"[Title/Abstract] OR "family assistance"[Title/Abstract] OR "social service"[Title/Abstract] OR "human service worker"[Title/Abstract] OR "human service workers"[Title/Abstract] OR "social care"[Title/ Abstract] OR "welfare work"[Title/Abstract] OR "welfare worker" [Title/Abstract] OR "social welfare" [Title/Abstract] OR "ambulatory youth welfare"[Title/Abstract] OR "social education work"[Title/Abstract] OR "social pedagogue $^{\prime}[$ Title/Abstract] OR "behavior specialist"[Title/ Abstract] OR "behavior specialists"[Title/Abstract] OR "behaviour specialist" [Title/Abstract] OR "behaviour specialists" [Title/Abstract] OR "educational guidance"[Title/ Abstract] OR Social workers[MeSH Terms] OR Social worker[MeSH Terms] OR Social work[MeSH Terms] OR Social welfare[MeSH Terms] OR social services[MeSH Terms]

Working condition "work conditions"[Title/Abstract] OR "working conditions"[Title/Abstract] OR "condition of work"[Title/Abstract] OR "occupational"[Title/Abstract] OR "occupation"[Title/Abstract] OR "workplace"[Title/ Abstract] OR "job conditions" [Title/Abstract] OR "working situation"[Title/Abstract] OR "work situtation"[Title/Abstract] OR "work environment" [Title/Abstract] OR "working environment"[Title/Abstract] OR "employment conditions"[Title/Abstract] OR "work characteristics"[Title/ Abstract] OR "workforce"[Title/Abstract] OR "work de-

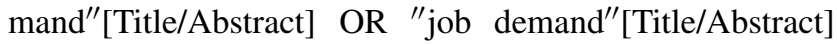
OR "work-related"[Title/Abstract] OR "worksite"[Title/ Abstract] OR "work site"[Title/Abstract] OR "workload"[Title/Abstract] OR "efford-reward"[Title/Abstract] OR "resource"[Title/Abstract] OR "resources"[Title/Abstract] OR "in-home work" [Title/Abstract] OR "in-home worker"[Title/Abstract] OR "work inside their clients home" [Title/Abstract] OR "work inside the clients home" [Title/Abstract] OR workplace[MeSH Terms] OR occupational exposure[MeSH Terms] OR workload[MeSH Terms]

Health Outcome "occupational health"[Title/Abstract] OR "well-being"[Title/Abstract] OR "wellbeing"[Title/Abstract] OR "strain"[Title/Abstract] OR "coping strategy"[Title/ Abstract] OR "coping strategies" [Title/Abstract] OR "stress" [Title/Abstract] OR "burnout"[Title/Abstract] OR "distress" [Title/Abstract] OR "emotional exhaustion"[Title/Abstract] OR "depersonalization" [Title/Abstract] OR "depersonalisa- tion" [Title/Abstract] OR "personal accomplishment"[Title/ Abstract] OR "cynicism"[Title/Abstract] OR "consequences"[Title/Abstract] OR "resilience"[Title/Abstract] OR "irritation"[Title/Abstract] OR "mental illness"[Title/ Abstract] OR "mental disease"[Title/Abstract] OR "job satisfaction" [Title/Abstract] OR "work satisfaction"[Title/ Abstract] OR "depression"[Title/Abstract] OR "fatigue" [Title/Abstract] OR "turnover intention"[Title/Abstract] OR "intention to leave" [Title/Abstract] OR "stress-related disorder" [Title/Abstract] OR "stress-related disorders" [Title/Abstract] OR "anxiety"[Title/Abstract] OR "anxieties" [Title/Abstract] OR "psychosomatic disorder"[Title/Abstract] OR "psychosomatic disorders"[Title/Abstract] OR "somatoform disorder"[Title/Abstract] OR "somatoform disorders"[Title/Abstract] OR "work engagement"[Title/ Abstract] OR "quality of life"[Title/Abstract] OR "worklife-balance"[Title/Abstract] OR "absenteeism" [Title/Abstract] OR "gratification"[Title/Abstract] OR burnout, professional[MeSH Terms] OR Adaptation, Psychological[MeSH Terms] OR occupational health[MeSH Terms] OR health status[MeSH Terms] OR Stress, Psychological[MeSH Terms] OR Mental Health[MeSH Terms] OR Depersonalization[MeSH Terms] OR Health Resources[MeSH Terms] OR Job Satisfaction[MeSH Terms] OR Occupational Diseases[MeSH Terms] OR Anxiety[MeSH Terms] OR Anxiety Disorders[MeSH Terms] OR Psychophysiologic Disorders[MeSH Terms] OR Somatoform Disorders[MeSH Terms] OR Occupational Stress[MeSH Terms] OR Quality of Life[MeSH Terms] OR Fatigue[MeSH Terms] OR Absenteeism[MeSH Terms]

\section{Suchstrategie in Psychlnfo (via ovid)}

1. Social Work (population)

2. Working condition (exposition)

3. Health Outcome

4. (1) AND (2) AND (3)

5. Limit 4 to Publication date from 2000 to 2019

\section{Social Work}

1. „social work“.ti OR „,social work“.ab OR "social worker".ti OR ,social work“.ab

2. “educational counselling”.ti OR ,educational counselling".ab

3. "youth work".ti OR "youth work".ab OR "youth worker".ti OR "youth worker".ab

4. "youth care".ti or "youth care".ab OR "youth service".ti OR "youth service".ab

5. "social service".ti OR "social service".ab OR "social care".ti OR "social care".ab

6. "outreach work".ti OR "outreach work".ab OR "outreach worker”.ti OR “outreach worker”.ab 
7. “community work".ti OR "community work".ab OR "community worker".ti OR "community worker".ab

8. “drug prevention".ti "drug prevention".ab

9. "family support".ti OR "family support".ab OR "familiy assistance".ti OR "familiy assistance". ab

10. "human service worker".ti OR" Human service worker".ab OR "human service workers".ti OR "human service workers".ab

11. "welfare work".ti OR "welfare work".ab OR "welfare worker".ti “welfare worker".ab

12. "social welfare".ti OR "social welfare".ab OR "ambulatory youth welfare".ti OR "ambulatory youth welfare".ab

13. "social education work".ti OR "social education work".ab

14. "social pedagogue".ti OR "social pedagogue".ab

15. "behaviour specialist".ti OR "behaviour specialist".ab OR "behavior specialist".ti OR behavior specialist".ab OR "behaviour specialists".ti OR "behaviour specialists".ab OR "behavior specialists".ti OR "behavior specialists".ab

16. „,educational guidance“.ti OR ,educational guidance“.ab

17. „social workers".mh

18. „welfare services“.mh

19. „welfare service“.mh

20. 1 or 2 or 3 or 4 or 5 or 6 or 7 or 8 or 9 or 10 or 11 or 12 or 13 or 14 or 15 or 16 or 17 or 18 or 19

\section{Working condition}

1. "work conditions".ti OR work conditions".ab OR "working conditions".ti OR "working conditions".ab OR "condition of work".ti OR "condition of work".ab OR "job conditions”.ti OR "job conditions".ab

2. "work situation".ti OR "work situation".ab OR "working situation".ti OR "working situation".ab

3. “occupational”.ti OR "occupational”.ab OR "occupation".ti OR "occupation".ab

4. “workplace”.ti OR "workplace”.ab

5. "work environment".ti OR "work environment".ab OR “working environment”.ti OR "working environment".ab

6. "employment conditions".ti OR "employment conditions".ab

1. "work characteristics".ti OR "work characteristics".ab

2. "workforce".ti OR "workforce".ab

3. “work demand".ti OR "work demand".ab OR "job demand".ti OR "job demand".ab

4. “work-related".ti OR "work-related".ab

5. “work site".ti OR “work site”.ab OR "worksite".ti OR "worksite".ab

6. “workload”.ti OR “workload”.ab
7. “efford-reward".to OR "efford-reward".ab

8. "resource".ti OR "resource".ab OR "resources".ti OR "resources".ab

9. "in-home work".ti OR "in-home work".ab OR "inhome worker".ti OR "in-home worker".ab

10. "work inside their clients home".ti OR "work inside their clients home".ab OR "work inside the clients home".ti OR "work inside the clients home".ab

11. "occupational exposure".mh

12. "workload".mh

13. “working conditions".mh

14. 1 or 2 or 3 or 4 or 5 or 6 or 7 or 8 or 9 or 10 or 11 or 12 or 13

\section{Health Outcome}

1. „occupational health“.ti OR ,occupational health“.ab

2. "well-being".ti OR "well-being".ab OR "wellbeing”.ti OR "wellbeing".ab

3. “strain".ti OR "strain".ab

4. “coping strategy".ti OR "coping strategy".ab OR "coping strategies".ti OR "coping strategies".ab

5. "stress".ti OR “stress".ab OR “distress".ti OR “distress".ab

6. "burnout".ti OR "burnout".ab

7. “emotional exhaustion”.ti OR "emotional exhaustion".ab

8. “depersonalization”.ti OR "depersonalization".ab OR “depersonalisation".ti OR “depersonalisation".ab

9. "personal accomplishment”.ti OR "personal accomplishment".ab

10. “cynicism”.ti OR “cynicism”.ab

11. "consequences".ti OR "consequences".ab

12. „resilience“.ti OR ,resilience“.ab

13. „,irritation“.ti OR ,,irritation“.ab

14. „mental illness“.ti OR ,mental illness“.ab OR “mental disease".ti OR "mental disease".ab

15. "job satisfaction". ti OR "job satisfaction".ab OR "work satisfaction".ti OR "work satisfaction".ab

16. “depression".ti OR "depression".ab

17. "fatigue".ti OR"fatigue".ab

18. "turnover intention".ti OR "turnover intention".ab OR "intention to leave".ti OR intention to leave".ab

19. "stress-related disorder".ti OR "stress-related disorder".ab OR "stress-related disorders".ti OR "stressrelated disorders".ab

20. "anxiety”.ti OR “anxiety”.ab OR “anxieties”.ti OR anxieties".ab

21. "psychosomatic disorder".ti OR psychosomatic disorder".ab OR "psychosomatic disorders".ti OR "psychosomatic disorders".ab 
22. "somatoform disorder".ti OR "somatoform disorder".ab OR "somatoform disorders".ti OR "somatoform disorders".ab

23. „work engagement”.ti OR, ,work engagement”.ab

24. "quality of life".ti OR "quality of life".ab

25. "work-life-balance".ti OR "work-life-balance".ab

26. “absenteeism".ti OR "absenteeism".ab

27. “gratification”.ti OR "gratification”.ab

28. "stress an d coping measures".mh

29. “coping behaviour".mh

30. “occupational health".mh

31. "stress".mh

32. "psychological stress".mh

33. "distress".mh

34. "mental health".mh

35. “depersonalisation”.mh

36. “job satisfaction”.mh"

37. "anxiety”.mh

38. “anxiety disorder”.mh

39. "somatoform disorders".mh

40. “well-being”.mh

41. "occupational stress".mh

42. "quality of life".mh

43. "fatigue".mh

44. "absentism".mh

1. 1 or 2 or 3 or 4 or 5 or 6 or 7 or 8 or 9 or 10 or 11 or 12 or 13 or 14 or 15 or 16 or 17 or 18 or 19 or 20 or 21 or 22 or 23 or 24 or 25 or 26 or 27 or 28 or 29 or 30 or 31 or 32 or 33 or 34 or 35 or 36 or 37 or 38 or 39 or 40 or 41 or 42 or 43 or 44

\section{Suchstrategie in Psyndex via PubPsych}

1. Social Work (population)

2. Working condition (exposition)

3. Health Outcome

4. 1 AND 2 AND 3

5. Limit 4 to publicated between 2000 and 2019

Social Work (ti="social work" OR ab="social work" OR ti="social worker" OR ab="social worker" OR ti="educational counselling" OR ab="educational counselling" OR ti=" youth work" OR ab="youth work" OR ti="youth worker" OR ab="youth worker" OR ti="youth care" OR $a b=$ "youth care" OR ti="youth service" OR ab="youth service" OR ti="outreach work" OR ab="outreach work" OR ti="outreach worker" OR ab="outreach worker" OR ti="community work" OR ab="community work" OR ti="community worker" OR ab="community worker" OR ti="drug prevention" OR ab="drug prevention" OR ti="family support" OR ab="family support" OR ti="familiy assistance" OR ab="familiy assistance" OR ti="social service" OR ab="social service" OR ti="Human service worker" OR ab="Human service worker" OR ti="Human service workers" OR ab="Human service workers" OR ti="social care" OR ab="social care" OR ti="welfare work" OR ab="welfare work" OR ti="welfare worker" OR $a b=$ "welfare worker" OR ti="social welfare" OR $a b=$ "social welfare" OR ti="ambulatory youth welfare" OR $a b=$ "ambulatory youth welfare" OR ti="social education work" OR ab="social education work" OR ti="social pedagogue" OR ab="social pedagogue" OR ti="behaviour specialist" OR $a b=$ "behaviour specialist" OR ti="behavior specialist" OR $a b=$ "behavior specialist" OR ti="behaviour specialists" OR ab="behaviour specialists" OR ti="behavior specialists" OR ab="behavior specialists" OR ti="educational guidance" OR ab="educational guidance" OR ct="social workers" OR ct="social work" OR ct="social welfare" OR ct="welfare services" OR ct="social casework" OR ct="social services")

Working condition (ti="work conditions" OR ab="work conditions" OR ti="working conditions" OR ab="working conditions" OR ti="work situation" OR ab="work situation" OR ti="working situation" OR ab="working situation" OR ti="condition of work" OR ab="condition of work" OR ti="occupational" OR ab="occupational" OR $\mathrm{ti}=$ "occupation" OR ab="occupation" OR ti="workplace" OR $a b=$ ="workplace" OR ti=" job conditions" OR ab="job conditions" OR ti="work environment" OR ab="work environment" OR ti="working environment" OR ab="working environment" OR ti="employment conditions" OR $\mathrm{ab}=$ "employment conditions" OR ti="work characteristics" OR ab="work characteristics" OR ti="workforce" OR $a b=$ "workforce" OR ti="work demand" OR ab="work demand" OR ti="job demand" OR ab="job demand" OR $\mathrm{ti}=$ "work-related" OR ab="work-related" OR ti="work site" OR ab="work site" OR ti="worksite" OR ab="worksite“" OR ti="workload" OR ab="workload" OR ti="effordreward" OR ab="efford-reward" OR ti="resource" OR $a b=$ "resource" OR ti="resources" OR ab="resources" OR $\mathrm{ti}=$ "in-home work" OR ab="in-home work" OR ti="inhome worker" OR ab="in-home worker" OR ti="work inside their clients home" OR ab="work inside their clients home" OR ti="work inside the clients home" OR $\mathrm{ab}=$ "work inside the clients home" OR ct="workplace" OR ct="occupational exposure" OR ct="workload" OR $\mathrm{ct}=$ "working condition")

Health Outcome (ti="occupational health" OR ab="occupational health" OR ti="well-being" OR ab="well-being" OR ti="wellbeing" OR ab="wellbeing" OR ti="strain" OR ab="strain" OR ti="coping strategy" OR ab="coping strategy" OR ti="coping strategies" OR ab="coping strategies" OR ti="stress OR ab="stress" OR ti="distress" OR ab="distress" OR ti="burnout" OR ab="burnout" OR 
ti="emotional exhaustion" OR ab="emotional exhaustion" OR ti="depersonalization" OR ab="depersonalization" OR ti="depersonalisation" OR ab="depersonalisation" OR ti="personal accomplishment" OR ab="personal accomplishment" OR ti="cynicism" OR ab="cynicism" OR ti="consequences" OR ab="consequences" OR ti="resilience" OR ab="resilience" OR ti="irritation" OR ab="irritation" OR ti="mental illness" $\mathrm{OR}$ ab="mental illness" OR ti="mental disease" OR ab="mental disease" OR ti=“job satisfaction" OR ab="job satisfaction" $O R$ ti="work satisfaction" OR ab="work satisfaction" OR ti="depression" OR ab="depression" OR ti="fatigue" OR $a b=$ "fatigue" OR ti= ,turnover intention“ $O R$ ab="turnover intention" OR ti="intention to leave" OR ab="intention to leave" OR ti="stress-related disorder" OR ab="stressrelated disorder" OR ti="stress-related disorders" OR $a b=$ "stress-related disorders" OR ti="anxiety" OR ab="anxiety" OR ti="anxieties" OR ab="anxietyies" OR ti="psychosomatic disorder" OR ab="psychosomatic disorder" OR ti="psychosomatic disorders" OR ab="psychosomatic disorders" OR ti="somatoform disorder" OR ab="somatoform disorder" OR ti="somatoform disorders" OR ab="somatoform disorders" OR ti="work engagement" OR ab="work engagement" OR ti="quality of life" OR ab="quality of life" OR ti="work-life-balance" OR ab="work-life-balance" OR ti="absenteeism" OR ab="absenteeism" OR ti="gratification" OR ab="gratification" OR ct="burnout" OR ct="adaption" OR ct="coping behaviour" OR ct="coping behaviour" OR ct="occupational health" OR ct= ,health status" OR ct="stress" OR ct="psychological stress" OR ct="distress" OR ct="mental health" OR ct="depersonalization" OR ct="health resources" OR ct="job satisfaction" OR ct="occupational diseases" OR ct="anxiety" OR ct="anxiety disorders" OR ct="psychosomatic disorders" OR ct="somatoform disorders" OR ct="well-being" OR ct="occupational stress" OR ct="quality of life" OR ct="fatigue" OR ct="absenteeims") 


\section{Suchbegriffe deutsch}

Tab. 2 Suchbegriffe deutsch

Table 2 Search terms german

\begin{tabular}{|c|c|c|}
\hline Population & Exposition & Outcome \\
\hline Sozialarbeiter* & $\begin{array}{l}\text { Arbeitsbedingungen, -situation, -an- } \\
\text { forderungen }\end{array}$ & $\begin{array}{l}\text { Gesundheitsstatus, -zustand, -folgen, gesundheit- } \\
\text { licher Zustand }\end{array}$ \\
\hline Soziale Arbeit, Sozialarbeit & Arbeitsbelastungen & Wohlbefinden, Wohlergehen \\
\hline In der sozialen Arbeit & Arbeit, Taetigkeit & Lebensqualität \\
\hline Sozialpaedagog* & Arbeitsbedingt* & Befinden, Befindensbeeinträchtigung \\
\hline Schulsozialarbeit & Beruflich* & Beanspruchung \\
\hline Streetwork* & $\begin{array}{l}\text { Arbeitsherausforderungen, Belas- } \\
\text { tungsfaktoren }\end{array}$ & Belastung*/-sfaktoren/-serleben \\
\hline Schulbegleitung & $\begin{array}{l}\text { Arbeitnehmer*, Mitarbeite*, Be- } \\
\text { schäftig* }\end{array}$ & Beschwerden \\
\hline Unterstützende Familienhilfe & Arbeitsplatz & Stress, Disstress \\
\hline Sozialpädagogische Familienhilfe & Anforderung & Auswirkungen \\
\hline (Ambulante) Jugendhilfe, Jugendhilfetraeger & Arbeitsmenge, Workload & Psychische Erkrankung* \\
\hline Erziehungsberatung & Ressource, Ressourcen & $\begin{array}{l}\text { Burnout, Depersonalisierung, Emotionale Er- } \\
\text { schöpfung }\end{array}$ \\
\hline Kinder- und Jugendarbeit & Aufsuchende (Sozial)Arbeit & Depression \\
\hline Erziehungsberatung & Beruf, Job & Zynismus \\
\hline Kinder- und Jugendarbeit & Gratifikatio* & Schlafstoerung, Muedigkeit \\
\hline $\begin{array}{l}\text { Allgemeiner sozialer Dienst, ASD, Sozial* } \\
\text { Dienst* }\end{array}$ & Kontroll* & Irritation, Reizbarkeit \\
\hline $\begin{array}{l}\text { Jugendamt/Jugendaemter, Amt fuer Jugend und } \\
\text { Familie }\end{array}$ & Stressor, Stressfaktor & Bewältigungsstrategien, Coping \\
\hline Jugendsozialarbeit, Jugendarbeit, Jugendverband & & Arbeitszufriedenheit \\
\hline $\begin{array}{l}\text { Kinder- und Familienzentren, Familientreff, } \\
\text { Kinderschutzzentr* }\end{array}$ & & Krank(heit) \\
\hline Kirchliche Beratungsstellen & & Überforderung, Limitation \\
\hline Offene Kinder- und Jugendarbeit & & Arbeitsgesundheit \\
\hline Jugend(hilfe)zentrum, Jugendintegrationsdienst & & Leistungsfähigkeit \\
\hline Mobile Jugendarbeit & & Beruf aufgeben/wechseln/verlassen, Fluktuation \\
\hline Soziale Einrichtungen & & (Arbeits)engagement, (Arbeits)motivation \\
\hline Jugendberufshilfe & & Resilienz, Work-Life-Balance \\
\hline \multicolumn{3}{|l|}{ Sozialpaedagogische Fachkräfte } \\
\hline \multicolumn{3}{|l|}{ Erziehungsbeistand, Erziehungshilfe } \\
\hline \multicolumn{3}{|l|}{ Sucht- und Drogenpraevention, Suchtpraevenion } \\
\hline \multicolumn{3}{|l|}{$\begin{array}{l}\text { Erziehung in einer Tagesgruppe, soziale Grup- } \\
\text { penarbeit }\end{array}$} \\
\hline \multicolumn{3}{|l|}{ Intensive sozialpaedagogische Einzelbetreuung } \\
\hline \multicolumn{3}{|l|}{ Kinder- und Jugendanwaltschaft } \\
\hline \multicolumn{3}{|l|}{ Vormundschaft } \\
\hline \multicolumn{3}{|l|}{ Interventionsstelle fuer Gewalt in der Familie } \\
\hline Familienmediation & & \\
\hline
\end{tabular}




\section{Anlage 2}

Tab. 3 Exzerpt der Studien Table 3 Excerpt of the studies

\begin{tabular}{|c|c|c|c|c|}
\hline $\begin{array}{l}\text { Autor, Jahr, } \\
\text { Land }\end{array}$ & Methode & Stichprobe, Zielgruppe & Erhobene Expositionen/Arbeitsanforderung & Erhobener Outcome \\
\hline $\begin{array}{l}\text { Antonopoulou } \\
\text { et al. (2017) } \\
\text { (UK) }\end{array}$ & $\begin{array}{l}\text { Quantitative } \\
\text { Querschnitt- } \\
\text { studie }\end{array}$ & $\begin{array}{l}N=193 \text { Kinderschüt- } \\
\text { zer*innen (child protecti- } \\
\text { on workers) }\end{array}$ & $\begin{array}{l}\text { Kleine Teams } \\
\text { Rekrutierung von gut ausgebildetem Personal } \\
\text { Klare Werte } \\
\text { Unterstützung der Organisation } \\
\text { Jobautonomie } \\
\text { Entschiedungsfreiheit } \\
\text { Jobperspektiven } \\
\text { Zufriedenheit } \\
\text { Arbeitsumgebung } \\
\text { Berufserfahrung }\end{array}$ & $\begin{array}{l}\text { Wohlbefinden bei der } \\
\text { Arbeit (Worker well- } \\
\text { being General Health } \\
\text { Questionnaire GHQ- } \\
12 \text { by Goldberg and } \\
\text { Williams 1988) }\end{array}$ \\
\hline $\begin{array}{l}\text { Barford } \\
\text { und Whel- } \\
\text { ton (2010) } \\
\text { (Kanada) }\end{array}$ & $\begin{array}{l}\text { Quantitative } \\
\text { Querschnitt- } \\
\text { studie }\end{array}$ & $\begin{array}{l}N=89 \\
\text { Staatliche und gemein- } \\
\text { nützige Einrichtungen } \\
\text { der Kinder- und Jugend- } \\
\text { hilfe (Government and } \\
\text { non-profit child and } \\
\text { youth care facilities) }\end{array}$ & $\begin{array}{l}\text { Arbeits-/Zeitdruck } \\
\text { Neurotizismus } \\
\text { Rollenklarheit } \\
\text { Engagement/Beteiligung } \\
\text { Unterstützung anderer } \\
\text { Verträglichkeit } \\
\text { Extravertiertheit } \\
\text { Gewissenhaftigkeit }\end{array}$ & $\begin{array}{l}\text { Burnout Komponenten: } \\
\text { Emotionale Erschöpfung, } \\
\text { Depersonalisierung, } \\
\text { persönliche Leistungsfä- } \\
\text { higkeit (Maslach Burnout } \\
\text { Inventory-Human Ser- } \\
\text { vices Survey (Maslach } \\
\text { et al. 1996)) }\end{array}$ \\
\hline $\begin{array}{l}\text { Beckmann } \\
\text { et al. (2009) } \\
\text { (Deutsch- } \\
\text { land, NRW) }\end{array}$ & $\begin{array}{l}\text { Quantitative } \\
\text { Querschnitt- } \\
\text { studie }\end{array}$ & $\begin{array}{l}N=261 \text { aus } 30 \text { Jugend- } \\
\text { hilfeeinrichtungen }\end{array}$ & $\begin{array}{l}\text { Handlungsspielräume } \\
\text { Fragmentierung der Arbeit } \\
\text { Rückmeldung durch die Leitung } \\
\text { Rückmeldung aus der Tätigkeit selbst } \\
\text { Professionelle Autonomie } \\
\text { Bedeutsamkeit der Tätigkeit } \\
\text { Wahrgenommene Vielfalt } \\
\text { Ganzheitlichkeit der Aufgabe }\end{array}$ & $\begin{array}{l}\text { Burnout nach Maslach } \\
\text { s. oben }\end{array}$ \\
\hline $\begin{array}{l}\text { Biggart } \\
\text { et al. (2017) } \\
\text { (UK) }\end{array}$ & $\begin{array}{l}\text { Qualitative } \\
\text { Studie } \\
\text { (halb-struk- } \\
\text { turierte, } \\
\text { leitfaden- } \\
\text { gestuitzte } \\
\text { Telefonin- } \\
\text { terviews) }\end{array}$ & $\begin{array}{l}n=52 \text { Familienhilfe des } \\
\text { Jugendamtes } \\
\text { (Frontline Child and Fa- } \\
\text { mily social worker from } \\
\text { Children's Service de- } \\
\text { partments within } 8 \text { local } \\
\text { authorities in England) }\end{array}$ & $\begin{array}{l}\text { Zu große Arbeitsmenge } \\
\text { Hohe Fluktuationen } \\
\text { Führungskräfte mit schlechter Emotionsregulie- } \\
\text { rung } \\
\text { Offen effizienzorientierte Führung } \\
\text { vertrauensvolles Verhältnis zur Führungskraft } \\
\text { Positives und konstruktives Feedback, Wertschät- } \\
\text { zung wenig Zeit für reflektive Supervisionen } \\
\text { Verfügbarkeit von Supervisoren } \\
\text { Soziale Unterstützung von Kollegen } \\
\text { Kooperation und Unterstützung im Team } \\
\text { Gefühl von Team-Zugehörigkeit } \\
\text { Zeit für (formellen und informellen) Austausch } \\
\text { Geregelter, regelmäßiger Kontakt zu Kollegen } \\
\text { Förderung des Selbstwertgefühls } \\
\text { Akzeptanz der eigenen Person }\end{array}$ & $\begin{array}{l}\text { Selbstwertgefühl, } \\
\text { psychische Gesundheit, } \\
\text { Burnout, Wohlbefinden, } \\
\text { Effektivität der Jobaus- } \\
\text { führung }\end{array}$ \\
\hline $\begin{array}{l}\text { Dagan et al. } \\
\text { (2016) } \\
\text { (Israel) }\end{array}$ & $\begin{array}{l}\text { Quantitative } \\
\text { Querschnitt- } \\
\text { studie }\end{array}$ & $\begin{array}{l}n=255 \\
\text { Kinderschutz, Sozialer } \\
\text { Dienst (124/48,6\% child } \\
\text { protection; } 131 / 51,4 \% \\
\text { social service workers: } \\
\text { from } 11 \text { social service } \\
\text { department in Israel) }\end{array}$ & $\begin{array}{l}\text { Rollenkonflikte } \\
\text { Traumatische Erfahrungen } \\
\text { Ausmaß der Exposition zu Kindermisshandlung } \\
\text { Selbstwirksamkeit } \\
\text { Empfundene soziale Unterstützung } \\
\text { Effektivität von Supervisionen }\end{array}$ & $\begin{array}{l}\text { Sekundärtraumatischer } \\
\text { Stress }\end{array}$ \\
\hline
\end{tabular}


Tab. 3 (Fortsetzung)

Table 3 (Continued)

\begin{tabular}{|c|c|c|c|c|}
\hline $\begin{array}{l}\text { Autor, Jahr, } \\
\text { Land }\end{array}$ & Methode & Stichprobe, Zielgruppe & Erhobene Expositionen/Arbeitsanforderung & Erhobener Outcome \\
\hline $\begin{array}{l}\text { Harker } \\
\text { et al. (2016) } \\
\text { (Australien, } \\
\text { Queensland) }\end{array}$ & $\begin{array}{l}\text { Quantitative } \\
\text { Querschnitt- } \\
\text { studie }\end{array}$ & $\begin{array}{l}N=133 \\
\text { Dienstleistungsberufe } \\
\text { in den Bereichen Psy- } \\
\text { chologie, Soziale Arbeit, } \\
\text { Beratung, Jugendhilfe } \\
\text { (human service profes- } \\
\text { sionals in the fields of } \\
\text { psychology, social work, } \\
\text { counselling, youth and } \\
\text { foster care work) }\end{array}$ & $\begin{array}{l}\text { Achtsamkeit } \\
\text { Resilienz (als Mediator) }\end{array}$ & $\begin{array}{l}\text { Disstress, Burnout (Är- } \\
\text { ger, Erschöpfung, Frus- } \\
\text { tration, Depressionen) } \\
\text { und Sekundärtraumati- } \\
\text { scher Stress (negative } \\
\text { Gefühle, Angst, arbeits- } \\
\text { bedingtes Trauma) } \\
\text { Berufliche Lebensqua- } \\
\text { lität Skala Version } 5 \\
\text { (ProQOL-5) }\end{array}$ \\
\hline $\begin{array}{l}\text { McFadden } \\
\text { et al. (2015) } \\
\text { (global) }\end{array}$ & $\begin{array}{l}\text { Systematisches } \\
\text { Review }\end{array}$ & 65 Artikel & $\begin{array}{l}\text { Persönliche Leidensgeschichte } \\
\text { Arbeitsmenge } \\
\text { Niedriges Gehalt und Vorteile (mangelnde Wert- } \\
\text { schätzung, mangelnder Einsatz der Organisation) } \\
\text { Aus- und Fortbildung } \\
\text { Aktive Coping-Strategien } \\
\text { Unterstützung von Kolleg*innen }\end{array}$ & $\begin{array}{l}\text { Selbstwertgefühl, Selbst- } \\
\text { wirksamkeit, Arbeits- } \\
\text { zufriedenheit, Intention } \\
\text { zu bleiben/zu gehen, } \\
\text { Burnout, Resilienz, } \\
\text { Sekundärtraumaitscher } \\
\text { Stress, Distress }\end{array}$ \\
\hline
\end{tabular}

\begin{tabular}{|c|c|c|c|c|}
\hline $\begin{array}{l}\text { McFadden } \\
\text { et al. (2018) } \\
\text { (Northern } \\
\text { Ireland) }\end{array}$ & $\begin{array}{l}\text { Quantitative } \\
\text { Querschnitt- } \\
\text { studie }\end{array}$ & $\begin{array}{l}N=162 \\
\text { Sozialarbeiter*innen } \\
\text { aus Gesundheits- und } \\
\text { Sozialzentren die mit } \\
\text { Familien }(56 \%) \text {, und } \\
\text { Ehrenamtlichen }(11 \%) \text {, } \\
\text { therapeutisch }(6 \%) \text { oder } \\
\text { im Kinderschutz (5\%) } \\
\text { arbeiten }\end{array}$ & $\begin{array}{l}\text { Resilienz (als Moderator für Arbeitsmenge, Kon- } \\
\text { trolle und Werteübereinstimmung) } \\
\text { Werteübereinstimmung } \\
\text { Arbeitsmenge } \\
\text { Gesellschaftliche Kontrolle } \\
\text { Fairness } \\
\text { Gehalt } \\
\text { Empfundene Anerkennung }\end{array}$ & $\begin{array}{l}\text { Burnout (Maslach Burn- } \\
\text { out inventory von Mas- } \\
\text { lach und Leiter 1986) }\end{array}$ \\
\hline $\begin{array}{l}\text { Poulsen } \\
(2014)+2016 \\
\text { (Deutschland) }\end{array}$ & $\begin{array}{l}\text { Qualitatives } \\
\text { Forschungs- } \\
\text { design } \\
\text { (schriftliche } \\
\text { Befragung) }\end{array}$ & $\begin{array}{l}N=100 \\
\text { Beschäftigte aus Ju- } \\
\text { gendämtern (27), offe- } \\
\text { ne Jugendarbeit (21), } \\
\text { betreute Wohnformen } \\
\text { (15), mobile Jugend- } \\
\text { arbeit/Streetwork (13), } \\
\text { Schulsozialarbeit (11), } \\
\text { Jugendberufshilfe (7), } \\
\text { Jugendberatung (6) }\end{array}$ & $\begin{array}{l}\text { Zeit- und Termindruck } \\
\text { Umständliche/lebensfremde Bürokratie } \\
\text { Personalmangel } \\
\text { Arbeitsverdichtung } \\
\text { Fehlende Kooperationsbereitschaft der Kli- } \\
\text { ent*innen } \\
\text { Rollenkonflikt } \\
\text { Hohe Verantwortung } \\
\text { nicht vorhersehbare Krisen } \\
\text { mangelnde Wertschätzung } \\
\text { Konflikte mit Vorgesetzten und im Team } \\
\text { Fehlende Rückendeckung durch Vorgesetzte } \\
\text { Überlastung der Kolleg*innen } \\
\text { eigene Grenzen nicht erkennen und setzen } \\
\text { Diskrepanz zwischen Leistbarem und Gefordertem } \\
\text { Erleben menschlicher Tragödien } \\
\text { Desinteresse der Politik } \\
\text { Unterstützend: } \\
\text { Supervisionen } \\
\text { kollegiale Beratung, Reflexion und Austausch im } \\
\text { Team } \\
\text { Fortbildungsmöglichkeiten, Gesundheitsförde- } \\
\text { rungsseminare } \\
\text { flexible Arbeitszeiten, } \\
\text { Gestaltungsspielraum }\end{array}$ & $\begin{array}{l}\text { Symptome: } \\
\text { Schlafstörungen, Müdig- } \\
\text { keit und Antriebslosig- } \\
\text { keit, Magen-, Rücken- } \\
\text { probleme, Kopfschmer- } \\
\text { zen/Migräne, Allgemeine } \\
\text { Erschöpfung, innere Un- } \\
\text { ruhe, Bluthochdruck, er- } \\
\text { höhte Infektanfälligkeit, } \\
\text { depressive Verstimmun- } \\
\text { gen, Burnout-Symptome } \\
\text { inkl. Panikattacken }\end{array}$ \\
\hline
\end{tabular}

Verbundenheit zur Führungskraft

Hohes Engagement

Betriebsklima und -kultur

Soziale Unterstützung und Supervisionen

Resilienz (als Moderator für Arbeitsmenge, Kontrolle und Werteübereinstimmung)

bereinstimmun

Fairness

Gehalt

Zeit- und Termindruck

Umständliche/lebensfremde Bürokratie

Fehlende Kooperationsbereitschaft der Klient*innen

Hohe Verantwortung

nicht vorhersehbare Krisen

mangelnde Wertschätzung

Konflikte mit Vorgesetzten und im Team

Überlastung der Kolleg*innen

Erleben menschlicher Tragödien

Unterstützend:

Supervisionen

Team

rungsseminare

Gestaltungsspielraum 
Tab. 3 (Fortsetzung)

Table 3 (Continued)

\begin{tabular}{|c|c|c|c|c|}
\hline $\begin{array}{l}\text { Autor, Jahr, } \\
\text { Land }\end{array}$ & Methode & Stichprobe, Zielgruppe & Erhobene Expositionen/Arbeitsanforderung & Erhobener Outcome \\
\hline $\begin{array}{l}\text { Winkens } \\
\text { (2016) } \\
\text { (Deutsch- } \\
\text { land) }\end{array}$ & $\begin{array}{l}\text { Qualitative } \\
\text { Experten- } \\
\text { interviews } \\
\text { (halbstruk- } \\
\text { turiert, } \\
\text { schriftlich) }\end{array}$ & $\begin{array}{l}N=5 \\
\text { Expert*innen Führungs- } \\
\text { kräfte in der Jugendhilfe }\end{array}$ & $\begin{array}{l}\text { Mangelnde personelle und finanzielle Ressourcen } \\
\text { (Diskrepanz zu vorliegenden Klientenbedarfen) } \\
\text { Direkter Kontakt zu hoch belasteten/ } \\
\text { traumatisierten Klient*innen } \\
\text { Risiko körperlicher Übergriffe } \\
\text { Kurzfristige Kontroll- und Hilfeansprüche durch } \\
\text { Politik und Verwaltung } \\
\text { Mangelnde Vorhersehbarkeit } \\
\text { Unterstützend: } \\
\text { Supervision stärkt die Resilienz, durch Förderung } \\
\text { von Selbstwirksamkeit, Kohärenzgefühl, soziale } \\
\text { Unterstützung, aktive Coping-Strategien } \\
\text { entwickeln von Handlungsalternativen } \\
\text { Bewältigen von Erfahrungen } \\
\text { Distanz, Schutz- und Rückzugsraum } \\
\text { Förderung des Kooperationsverhaltens im Team } \\
\text { Verantwortungsbereich abstecken } \\
\text { Austausch und soziale Unterstützung } \\
\text { Entscheidungsprozesse analysieren } \\
\text { Förderung protektiver Persönlichkeitsfaktoren } \\
\text { Verbesserung der Kommunikation }\end{array}$ & $\begin{array}{l}\text { Personale und organisa- } \\
\text { tionale Resilienz, } \\
\text { Psychische Widerstands- } \\
\text { fähigkeit der Beschäftig- } \\
\text { ten }\end{array}$ \\
\hline
\end{tabular}

Open Access Dieser Artikel wird unter der Creative Commons Namensnennung 4.0 International Lizenz veröffentlicht, welche die Nutzung, Vervielfältigung, Bearbeitung, Verbreitung und Wiedergabe in jeglichem Medium und Format erlaubt, sofern Sie den/die ursprünglichen Autor(en) und die Quelle ordnungsgemäß nennen, einen Link zur Creative Commons Lizenz beifügen und angeben, ob Änderungen vorgenommen wurden.

Die in diesem Artikel enthaltenen Bilder und sonstiges Drittmaterial unterliegen ebenfalls der genannten Creative Commons Lizenz, sofern sich aus der Abbildungslegende nichts anderes ergibt. Sofern das betreffende Material nicht unter der genannten Creative Commons Lizenz steht und die betreffende Handlung nicht nach gesetzlichen Vorschriften erlaubt ist, ist für die oben aufgeführten Weiterverwendungen des Materials die Einwilligung des jeweiligen Rechteinhabers einzuholen.

Weitere Details zur Lizenz entnehmen Sie bitte der Lizenzinformation auf http://creativecommons.org/licenses/by/4.0/deed.de.

\section{Literatur}

Allroggen M, Fegert JM, Rau T (2017) Psychische Belastung von Fachkräften in (sozial-) pädagogischen Arbeitsfeldern. Eine Übersichtsarbeit über Prävalenz, Entstehungsbedingungen, Folgen und Unterstützungsmöglichkeiten. Soz Extra 5:49-53

Antonopoulou P, Killian M, Forrester D (2017) Levels of stress and anxiety in child and family social work: Workers' perception of organisational structure, professional support and workplace opportunities in Children's Service in the UK. Child Youth Serv Rev 76:42-50

Backe EM, Seidler A, Latza U, Rossnagel K, Schumann B (2012) The role of psychosocial stress at work for the development of cardiovascular diseases: a systematic review. Int Arch Occup Environ Health 85:67-79

Baldschun A (2019) Arbeitszufriedenheit von Sozialarbeitern: Konzepte, Strukturen und Faktoren des beruflichen Wohlbefindens von Sozialarbeitern und Sozialarbeiterinnen in sozialen Diensten.
In: Borrmann S, Fedke C, Thiessen B (Hrsg) Soziale Kohäsion und gesellschaftliche Wandlungsprozesse. Sozialer Wandel und Kohäsionsforschung. Springer VS, Wiesbaden

Barford SW, Whelton WJ (2010) Understanding burnout in child and youth care. Child Youth Care Forum 39:271-287

BAuA (2018) Sicherheit und Gesundheit bei der Arbeit (SUGA) - Berichtsjahr 2018. Unfallverhütungsbericht Arbeit, 2. Aufl. Bundesministerium für Arbeit und Soziales (BMAS), Dortmund, Berlin, Dresden

Beck D, Berger S, Breutmann N, Fergen A, Gregersen S, Morschhäuser M, Reddehase B, Ruck YR, Sandrock S, Theiler A (2017ed) Arbeitsschutz in der Praxis. Empfehlungen zur Umsetzung der Gefährdungsbeurteilung psychischer Belastung. In: Gemeinsame Deutsche Arbeitsschutzstrategie-Arbeitsprogramm Psyche (Hrsg) Gemeinsame Deutsche Arbeitsschutzstrategie, 2017. Aufl. Bundesministerium für Arbeit und Soziales, Berlin (Referat IIIb2, 3)

Beckmann C, Maar K, Otto H-U, Schaarschuch A, Schrödter M (2009) Burnout als Folge restringierender Arbeitsbedingungen? Neue Prax 9:194-205 (Sonderheft)

Ben-Zur H, Michael K (2007) Burnout, social support, and coping at work among social workers, psychologists, and nurses: the role of challenge/control appraisals. Soc Work Health Care 45:63-82

Bettany-Saltikov J (2012) How to do a systematic literature review in nursing: a step-by-step guide. Open University Press, Maidenhead

Biggart L, Ward E, Cook L, Schofield G (2017) The team as a secure base: promoting resilience and competence in child and family social work. Child Youth Serv Rev 83:119-130

Bonde JP (2008) Psychosocial factors at work and risk of depression: a systematic review of the epidemiological evidence. Occup Environ Med 85:438-445

Bride BE, Robinson MM, Yegidis B, Figley CR (2004) Development and validation of the secondary traumatic stress scale. Res Soc Work Pract 14:27-35

Brodersen S, Lück P (2017) Erwerbstätigenbefragung zum Stellenwert der Arbeit. In: iga.Barometer 2016. Iga.Report, Bd. 36. Zukunft der Arbeit GmbH, Dresden

Statistisches Bundesamt (2018) Statistiken der Kinder- und Jugendhilfe. Einrichtungen und tätige Personen (ohne Tageseinrichtungen für Kinder), 2016 
Bundesministerium für Familie, Senioren, Frauen und Jugend (BMFSFJ) (2014) Kinder- und Jugendhilfe Achtes Buch Sozialgesetzbuch

CASP (2018) CASP qualitative checklist. https://casp-uk.net/wpcontent/uploads/2018/01/CASP-Qualitative-Checklist-2018.pdf. Zugegriffen: 15. Febr. 2019

da Costa BR, Vieira ER (2010) Risk factors for work-related musculoskeletal disorders: a systematic review of recent longitudinal studies. Am J Ind Med 53:285-323

Dagan SW, BEN-Porat A, Itzhaky H (2016) Child protection workers dealing with child abuse: the contribution of personal, social and organizational resources to secondary traumatization. Child Abuse Negl 51:203-211

DBSH, Deutscher Berufsverband für soziale Arbeit (2016) Deutschsprachige Definition Sozialer Arbeit. Fachbereichstag Soziale Arbeit und DBSH.

GDA (2017) Psychische Arbeitsbelastung und Gesundheit: Leitung des GDA-Arbeitsprogramms Psyche c/o Bundesministerium für Arbeit und Soziales, Referat IIIb2

Glaser J, Seubert C (2014) Arbeitsbezogene psychosoziale Determinanten von Gesundheit. Arbeitshandeln, Arbeitsbelastung, Arbeitsorganisation. In: Angerer P, Glaser J, Gündel H, Henningsen P, Lahmann C, Letzel S (Hrsg) Psychische und psychosomatische Gesundheit in der Arbeit. Wissenschaft, Erfahrung, Lösungen aus Arbeitsmedizin, Arbeitspsychologie und Psychosomatischer Medizin. ecomed Medizin, Heidelberg

Goldberg DP (1972) The detection of psychiatric illness by questionnaire. A technique for the identification and assessment of nonpsychotic psychiatric illness. Oxford University Press, London, New York

Goldberg DP, Williams P (1988) A user's guide to the General Health Questionnaire. nferNelson, Windsor

Grebner S, Berlowitz I, Alvarado V, Cassina M (2010) Stressstudie 2010 Stress bei Schweizer Erwerbstätigen Zusammenhänge zwischen Arbeitsbedingungen, Personenmerkmalen, Befinden und Gesundheit. BBL, Bern

Grobe T, Bessel S (2020) Gesundheitsreport - Arbeitsunfähigkeiten. aQua - Institut für angewandte Qualitätsförderung und Forschung im Gesundheitswesen, Hamburg

Gusy B (2017) Arbeit und Gesundheit: Eine metaanalytische Befundintegration. Habilitation, Freien Universität Berlin

Harker R, Pidgeon AM, Klaassen F, King S (2016) Exploring resilience and mindfulness as preventative factors for psychological distress burnout and secondary traumatic stress among human service professionals. Work 54:631-637

Hasenfeld Y (2010) Organizational responses to social policy: the case of welfare reform. Adm Soc Work 34:148-167

Hussein S (2018) Work engagement, burnout and personal accomplishments among social workers: a comparison between those working in children and adults' services in England. Adm Policy Ment Health 45:911-923

Jacobs B (2008) Übereinstimmungsmaße für zwei Rater bei mehreren Merkmalsausprägungen. http://bildungswissenschaften.unisaarland.de/personal/jacobs/diagnostik/objektiv/uebereinstimmung. html. Zugegriffen: 4. Apr. 2019

Johnson J, Hall LH, Berzins K, Baker J, Melling K, Thompson C (2018) Mental healthcare staff well-being and burnout: a narrative review of trends, causes, implications, and recommendations for future interventions. Int J Mental Health Nurs 27:20-32

Kim H, Ji J, Kao D (2011) Burnout and physical health among social workers: a three-year longitudinal study. Social Work 56:258-268

Kramer I, Oster S, Blum M, Brodersen S, Cosmar M, Felfe J, Giesert M, Kratzer N, Kummer A, Liebrich A, Lück P, Pangert B, Reuter T, Schneberger T, Schuster S, Winterstein S (2015) Führungskräfte sensibilisieren und Gesundheit fördern - Ergebnisse aus dem Projekt "iga.Radar". BKK Dachverband, der Deutschen Gesetzlichen Unfallversicherung (DGUV), dem AOK-
Bundesverband und dem Verband der Ersatzkassen e. V. (vdek), Berlin

Krüger D, Parchmann I, Schecker H (2014) Methoden der naturwissenschaftsdidaktischen Forschung. Springer, Berlin, Heidelberg, New York

Lesener T, Gusy B, Wolter C (2019) The job demandsresources model: a meta-analytic review of longitudinal studies. Work Stress 33:76-103

Lloyd C, King R, Chenoweth L (2002) Social work, stress and burnout: a review. J Ment Health 11:255-266

Lohmann-Haislah A (2012) Stress aktuell - Ergebnisse der Erwerbstätigenbefragung. In: Stressreport Deutschland 2012. Psychische Anforderungen, Ressourcen und Befinden. Bonifatius $\mathrm{GmbH}$, Dortmund, Berlin, Dresden

Maslach C, Jackson SE (1981) The measurement of experienced burnout. J Organiz Behav 2:99-113

Maslach C, Jackson SE (1986) Maslach burnout inventory manual (2nd ed.). Consulting Psychologists Press, Palo Alto, CA

Maslach C, Jackson SE, Leiter MP (1996) Maslach Burnout Inventory. (3rd ed.). Consulting Psychologists Press, Palo Alto, CA

McFadden P, Campbell A, Taylor B (2015) Resilience and burnout in child protection social work: individual and organisational themes from a systematic literature review. Br J Soc Work 45:1546-1563

McFadden P, Mallet J, Leiter M (2018) Extending the two-process model of burnout in child protection workers: The role of resilience in mediating burnout via organizational factors of control, values, fairness, reward, workload, and community relationships. Stress Health 34:1-12

Mette J, Robelski S, Wirth T, Nienhaus A, Harth V, MacHe S (2020) Engaged, burned out, or both? A structural equation model testing work-related risk and protective factors for social workers serving refugees and homeless individuals. Int $\mathbf{J}$ Environ Res Public Health 17:583

Moher D, Liberati A, Tetzlaff J, Altman DG, Group TP (2009) Preferred reporting items for systematic reviews and meta-analyses: the Prisma statement. PLoS Med 6:e1000097

Nieuwenhuijsen K, Bruinvels D, Frings-Dresen M (2010) Psychosocial work environment and stress-related disorders, a systematic review. Occup Med (Lond) 60:277-286

Nüsken D (2020) Erziehungshilfen als Beruf - Einblicke in die Belastungen und Entlastungen eines Arbeitsfeldes. Springer VS, Wiesbaden

Oechler M (2009) Soziale Arbeit als Dienstleistung. In: Dienstleistungsqualität in der Sozialen Arbeit. VS, Wiesbaden

Paridon H, Mühlbach J (2016) Psychische Belastung in der Arbeitswelt - Eine Literaturanalyse zu Zusammenhängen mit Gesundheit und Leistung. IGA Report, Bd. 32. AOK-Bundesverband, BKK Dachverband e. V., Deutsche Gesetzliche Unfallversicherung (DGUV), Verband der Ersatzkassen e. V. (vdek), Berlin

Poulsen I (2014) Angst, man kommt zu spät und ein Kind ist tot" Stress und Belastungsfaktoren in der Jugendhilfe. Kinder Jugendschutz Wissensch Prax 59:121-127

Rau R, Henkel D (2013) Zusammenhang von Arbeitsbelastungen und psychischen Erkrankungen. Review der Datenlage. Nervenarzt 84:791-798

Rau R, Morling K, Rösler U (2010) Is there a relationship between major depression and both objective assessed and perceived job demand and job control? Work Stress 24:1-18

Rose M (2003) Good deal, bad deal? Job satisfaction in occupations. Work Employ Soc 17:503-530

Rothe I, Adolph L, Beermann B, Schütte M, Windel A, Grewer A, Lenhardt U, Michel J, Thomson B, Formazin M (2017) Psychische Gesundheit in der Arbeitswelt - Wissenschaftliche Standortbestimmung, 1. Aufl., Druck \& Verlag Kettler GmbH, Bönen

Scheu B, Autrata O (2018) Einführung. In: Scheu B, Autrata O (Hrsg) Das Soziale. Forschung, Innovation und Soziale Arbeit. Springer VS, Wiesbaden 
Schütte M, Windel A (2017) Psychische Gesundheit in der Arbeitswelt - Wissenschaftliche Standortbestimmung. Z Arb Wiss 71:1-5

Schwartz S L 2007 Engaging our workforce: how job demands and resources contribute to social worker burnout, engagement and intent to leave. Doctor of Philosophy (Ph.D.) in Social Work and Social Research Dissertation, Portland State University

Skirrow P, Hatton C (2007) 'Burnout' amongst direct care workers in services for adults with intellectual disabilities: a systematic review of research findings and initial normative data. J Appl Res Int Dis 20:131-144

Steinlin C, Dölitzsch C, Fischer S, Schmeck K, Fegert JM, Schmid M (2016) Der Zusammenhang zwischen Burnout-Symptomatik und Arbeitszufriedenheit bei pädagogischen Mitarbeitenden in der stationären Kinder- und Jugendhilfe. Prax Kinderpsychol Kinderpsychiatr 65:162-180

Vandenbroucke JP, Von Elm E, Altman DG, Gotzsche PC, Mulrow CD, Pocock SJ, Poole C, Schlesselman JJ, Egger M, Initiative S (2007) Strengthening the reporting of observational studies in epidemi- ology (strobe): explanation and elaboration. Ann Intern Med 147:W163-W194

Winkens H (2016) Der Beitrag von Supervision zur Stärkung von Resilienz in der Jugendhilfe. Eine empirische Untersuchung der Risikofaktoren für Arbeitnehmer/-innen und Organisationen der Jugendhilfe, sowie der Steigerung psychischer Widerstandsfähigkeit durch Supervision. Kassel University Press, Kassel

Wirth T, Wendeler D, Dulon M, Nienhaus A (2019) Sick leave and work-related accidents of social workers in Germany: an analysis of routine data. Int Arch Occup Environ Health 92:175-184

Wooten NR, Kim HJ, Fakunmoju SB (2011) Occupational stress in social work practice. In: Langan J, Cooper C (Hrsg) Handbook of stress in the occupations. Edward Elgar, Cheltenham 